Research Article

\title{
On Strongly Generalized Preinvex Fuzzy Mappings
}

\author{
Peide Liu, ${ }^{1}$ Muhammad Bilal Khan $\left(\mathbb{D},{ }^{2}\right.$ Muhammad Aslam Noor, ${ }^{2}$ \\ and Khalida Inayat Noor ${ }^{2}$ \\ ${ }^{1}$ School of Management Science and Engineering, Shandong University of Finance and Economics, Jinan 250014, Shandong, \\ China \\ ${ }^{2}$ Department of Mathematics, COMSATS University Islamabad, Islamabad, Pakistan
}

Correspondence should be addressed to Muhammad Bilal Khan; bilal42742@gmail.com

Received 14 December 2020; Revised 12 March 2021; Accepted 15 March 2021; Published 1 April 2021

Academic Editor: Kaleem R. Kazmi

Copyright (c) 2021 Peide Liu et al. This is an open access article distributed under the Creative Commons Attribution License, which permits unrestricted use, distribution, and reproduction in any medium, provided the original work is properly cited.

In this article, we introduce a new notion of generalized convex fuzzy mapping known as strongly generalized preinvex fuzzy mapping on the invex set. Firstly, we have investigated some properties of strongly generalized preinvex fuzzy mapping. In particular, we establish the equivalence among the strongly generalized preinvex fuzzy mapping, strongly generalized invex fuzzy mapping, and strongly generalized monotonicity. We also prove that the optimality conditions for the sum of G-differentiable preinvex fuzzy mappings and non-G-differentiable strongly generalized preinvex fuzzy mappings can be characterized by strongly generalized fuzzy mixed variational-like inequalities, which can be viewed as a novel and innovative application. Several special cases are discussed. Results obtained in this paper can be viewed as improvement and refinement of previously known results.

\section{Introduction}

Convexity plays an essential role in many areas of mathematical analysis and, due to its vast applications in diverse areas, many authors extensively generalized and extended this concept using novel and different approaches. In [1], Hasnon introduced a useful generalization of convex function that is an invex function and proved the validity of significant results that hold for both convex and invex functions under few conditions. Ben-Israel and Mond [2] introduced the concept of preinvex functions on the invex sets. Later on, Mohan and Neogy [3] further extended their work by proving that, subject to certain conditions, a preinvex function defined on the invex set is an invex function and vice versa and they also showed that quasi-invex function is quasi-preinvex function. Noor [4] and Weir and Mond [5] showed that the preinvex functions preserved some important properties of convex functions. Furthermore, Noor et al. [6-8] studied the optimality conditions of differentiable preinvex functions on the invex set that can be characterized by variational inequalities. Similarly, an important and significant generalization of convex function is strongly convex function, which is introduced by Polyak [9], which plays major role in optimization theory and related areas. Karmardian [10] discussed the unique existence of a solution of the nonlinear complementarity problems by using strongly convex functions. With the support of strongly convex functions, $\mathrm{Qu}$ and $\mathrm{Li}$ [11] and Nikodem and Pales [12] investigated the convergence analysis for solving equilibrium problems and variational inequalities. For further study, we refer the readers to [13-23] and the references therein for applications and properties of the strongly convex and preinvex functions.

In [24], the enormous research work fuzzy set and system has been dedicated to development of different fields and it plays an important role in the study of a wide class of problems arising in pure mathematics and applied sciences including operation research, computer science, managements sciences, artificial intelligence, control engineering, and decision sciences. The convex analysis has played an important and fundamental part in development of various fields of applied and pure science. Similarly, fuzzy convex analysis is used as the fundamental theory in fuzzy optimization. Fuzzy convex sets have been widely discussed by 
many authors, Liu [25] investigated some properties of convex fuzzy sets and, with the support of examples, he modified the concept of Zadeh [24] about shadow of fuzzy sets. Lowen [26] gathered some elementary well-known results about convex sets and proved separation theorem for convex fuzzy sets. Ammar and Metz [27] studied different types of convexity and defined generalized convexity of fuzzy sets. Furthermore, they formulated a general fuzzy nonlinear programing problem with application of the concept of convexity. In addition, the properties of convex fuzzy sets attracted the attention of a wide range of authors in $[3,28,29]$ and the references therein. To discuss fuzzy number, it is a generalized form of an interval (in crisp set theory). Zadeh [24] defined fuzzy numbers and then Dubois and Prade [30] extended the work of Zadeh using new conditions on fuzzy number. Further, Goetschel and Voxman [31] modified different condition on fuzzy numbers so that fuzzy numbers can be easily handled. For instance, in [30], on fuzzy number, one condition is that this is a continuous function but, in [31], fuzzy number is upper semicontinuous. The goal is that, by the relaxation of conditions on fuzzy number, we can easily define a metric for collection of fuzzy numbers; then this metric allows us to study some basic properties of topological space. Furukawa [32], Nanda and Kar [33], and Syau [34] worked on the concept of fuzzy mapping from $\mathbb{R}^{n}$ to the set of fuzzy numbers, Lipschitz continuity of fuzzy mapping, and fuzzy logarithmic convex and quasi-convex fuzzy mappings. Based on the concept of ordering illustrated by Goetschel and Voxman [35], Yan and Xu [36] presented the concepts of epigraphs and convexity of fuzzy mappings and described the characteristics of convex fuzzy and quasi-convex fuzzy mappings. The idea of fuzzy convexity has been generalized and extended in diversity of directions, which has significant implementation in many areas. It is worth mentioning that one of the most considered generalizations of convex fuzzy mapping is preinvex fuzzy mapping. The idea of fuzzy preinvex mapping on the fuzzy invex set was introduced and studied by Noor [37] and they verified that fuzzy optimality conditions of differentiable fuzzy preinvex mappings can be distinguished by variational-like inequalities. Moreover, any local minimum of a preinvex fuzzy mapping is a global minimum on invex set and a necessary and sufficient condition for fuzzy mapping is to be preinvex if its epigraph is an invex set. In [38], Syau further modified the concept of preinvex fuzzy mapping that was presented by Noor [37]. Syau and Lee [39] also discussed the terminologies of continuity and convexity through linear ordering and metric defined on fuzzy numbers. Extension in the Weierstrass Theorem from real-valued functions to fuzzy mappings is also one of their significant contributions in the literature. For recent applications, see [40-43] and the references therein.

Motivated and inspired by the ongoing research work and by the importance of the idea of invexity and preinvexity of fuzzy mappings, the paper is organized as follows. Section 2 recalls some basic definitions, preliminary notations, and results which will be helpful for further study. Section 3 introduces the notions of strongly generalized preinvex, quasi-preinvex, and log-preinvex fuzzy mappings and investigates some properties. Section 4 studies the new relationships among various concepts of strongly preinvex fuzzy mappings and establishes the equivalence among the strongly generalized preinvex fuzzy mapping, strongly generalized invex fuzzy mapping, and strongly generalized monotonicity. We also introduce several new concepts of strongly generalized invex fuzzy mappings and strongly generalized monotonicities and then discuss their relation. Section 5 introduces the new class of fuzzy variational-like inequality, which is known as strongly generalized fuzzy mixed variational-like inequality. Several special cases are discussed. This inequality is itself an interesting outcome of our main results.

\section{Preliminaries}

Let $\mathbb{R}$ be the set of real numbers. A fuzzy subset $A$ of $\mathbb{R}$ is characterized by a mapping $\psi: \mathbb{R} \longrightarrow[0,1]$ called the membership function, for each fuzzy set and $\gamma \in(0,1]$; then $\gamma$-level sets of $\psi$ are denoted and defined as follows: $\psi_{\gamma}=\{u \in \mathbb{R} \mid \psi(u) \geq \gamma\}$. If $\gamma=0$, then $\sup p(\psi)=\{u \in \mathbb{R} \mid$ $\psi(u)>0\}$ is called support of $\psi$. By $[\psi]^{0}$, we define the closure of $\sup p(\psi)$.

Definition 1. A fuzzy set is said to be fuzzy number with the following properties:

(1) $\psi$ is normal; that is, there exists $u \in \mathbb{R}$ such that $\psi(u)=1$

(2) $\varphi$ is upper semicontinuous; that is, for given $u \in \mathbb{R}$, there exist $\varepsilon>0$ and $\delta>0$ such that $\varphi(u)-\varphi(\vartheta)<\varepsilon$ for all $\vartheta \in \mathbb{R}$ with $|u-\vartheta|<\delta$

(3) $\psi$ is fuzzy convex; that is, $\psi((1-\tau) u+\tau \vartheta) \geq \min (\psi(u), \psi(\vartheta)) \quad \forall u, \vartheta \in \mathbb{R}$, $\tau \in[0,1]$

(4) $[\psi]^{0}$ is compact

$\mathbb{F}_{0}$ denotes the set of all fuzzy numbers. For fuzzy number, it is convenient to distinguish following $\gamma$-levels:

$$
\psi_{\gamma}=\{u \in \mathbb{R} \mid \psi(u) \geq \gamma\} .
$$

From these definitions, we have

$$
\psi_{\gamma}=\left[\psi_{*}(\gamma), \psi^{*}(\gamma)\right]
$$

where

$$
\begin{aligned}
& \psi_{*}(\gamma)=\inf \{u \in \mathbb{R} \mid \psi(u) \geq \gamma\}, \\
& \psi^{*}(\gamma)=\sup \{u \in \mathbb{R} \mid \psi(u) \geq \gamma\} .
\end{aligned}
$$

Each $\rho \in \mathbb{R}$ is also a fuzzy number, defined as

$$
\tilde{\rho}(u)= \begin{cases}1, & \text { if } u=\rho, \\ 0, & \text { if } u \neq \rho .\end{cases}
$$

Thus, a fuzzy number $\psi$ can be identified by a parametrized triple:

$$
\left\{\left(\psi_{*}(\gamma), \psi^{*}(\gamma), \gamma\right): \gamma \in[0,1]\right\} .
$$


This leads to the following characterization of a fuzzy number in terms of the two end point functions $\psi_{*}(\gamma)$ and $\psi^{*}(\gamma)$.

Theorem 1 (see [35]). Suppose that $\psi_{*}(\gamma):[0,1] \longrightarrow \mathbb{R}$ and $\psi^{*}(\gamma):[0,1] \longrightarrow \mathbb{R}$ satisfy the following conditions:

(1) $\psi_{*}(\gamma)$ is a nondecreasing function

(2) $\psi^{*}(\gamma)$ is a nonincreasing function

(3) $\psi_{*}(1) \leq \psi^{*}(1)$

(4) $\psi_{*}(\gamma)$ and $\psi^{*}(\gamma)$ are bounded and left continuous on $(0,1]$ and right continuous at $\gamma=0$

Then $\psi: \mathbb{R} \longrightarrow[0,1]$, defined by

$$
\psi(u)=\sup \left\{\gamma: \psi_{*}(\gamma) \leq u \leq \psi^{*}(\gamma)\right\}
$$

is a fuzzy number and its parameterization form is given by $\left\{\left(\psi_{*}(\gamma), \psi^{*}(\gamma), \gamma\right): \gamma \in[0,1]\right\}$. Moreover, if $\psi: \mathbb{R} \longrightarrow[0,1]$ is a fuzzy number with parametrization given by $\left\{\left(\psi_{*}(\gamma), \psi^{*}(\gamma), \gamma\right): \gamma \in[0,1]\right\}$, then functions $\psi_{*}(\gamma)$ and $\psi^{*}(\gamma)$ find conditions (1)-(4).

Let $\quad \psi, \phi \in \mathbb{F}_{0} \quad$ represent parametrically $\left\{\left(\psi_{*}(\gamma)\right.\right.$, $\left.\left.\psi^{*}(\gamma), \gamma\right): \gamma \in[0,1]\right\}$ and $\left\{\left(\phi(\gamma), \phi^{*}(\gamma), \gamma\right): \gamma \in[0,1]\right\}$, respectively. We say that $\psi^{*} \phi$ if, for all $\gamma \in(0,1]$, $\psi^{*}(\gamma) \leq \phi^{*}(\gamma)$ and $\psi_{*}(\gamma) \leq \phi_{*}(\gamma)$. If $\psi^{*} \phi$, then there exists $\gamma \in(0,1]$ such that $\psi^{*}(\gamma)<\phi^{*}(\gamma)$ or $\psi_{*}(\gamma) \leq \phi_{*}(\gamma)$. We say that it is comparable if, for any $\psi, \phi \in \mathbb{F}_{0}$, we have $\psi \leq \phi$ or $\psi \geq \phi$; otherwise, they are noncomparable. Sometimes we may write $\psi \leq \phi$ instead of $\psi \geq \phi$ and note that we may say that $\mathbb{F}_{0}$ is a partial ordered set under the relation $\leq$.

If $\psi, \phi \in \mathbb{F}_{0}$, there exists $\mu \in \mathbb{F}_{0}$ such that $\psi=\phi \tilde{+} \mu$, and then by this result we have the existence of Hukuhara difference of $\psi$ and $\phi$, and we say that $\mu$ is the H-difference of $\psi$ and $\phi$, denoted by $\psi-\phi$; see [43]. If $H$-difference exists, then

$$
\begin{aligned}
(\mu)^{*}(\gamma) & =(\psi \tilde{\sim} \phi)^{*}(\gamma)=\psi^{v}(\gamma)-\phi^{*}(\gamma), \\
(\mu)_{*} & =(\psi \tilde{-} \phi)_{*}(\gamma)=\psi_{*}(\gamma)-\phi_{*}(\gamma) .
\end{aligned}
$$

Now we discuss some properties of fuzzy numbers under addition and scaler multiplication; if $\psi, \phi \in \mathbb{F}_{0}$ and $\rho \in \mathbb{R}$, then $\psi \tilde{+} \phi$ and $\rho \psi$ are defined as

$$
\begin{aligned}
\psi \tilde{+} \phi & =\left\{\left(\psi_{*}(\gamma)+\phi_{*}(\gamma), \psi^{*}(\gamma)+\phi^{*}(\gamma), \gamma\right): \gamma \in[0,1]\right\}, \\
\rho \psi & =\left\{\left(\rho \psi_{*}(\gamma), \rho \psi^{*}(\gamma), \gamma\right): \gamma \in[0,1]\right\} .
\end{aligned}
$$

Remark 1. Obviously, $\mathbb{F}_{0}$ is closed under addition and nonnegative scaler multiplication and the above-defined properties on $\mathbb{F}_{0}$ are equivalent to those derived from the usual extension principle. Furthermore, for each scalar number $\rho \in \mathbb{R}$,

$$
\tilde{\psi+\rho}=\left\{\left(\psi_{*}(\gamma)+\rho, \psi^{*}(\gamma)+\rho, \gamma\right): \gamma \in[0,1]\right\} .
$$

Definition 2. A mapping $\mathscr{T}: \gamma \longrightarrow \mathbb{F}_{0}$ is called fuzzy mapping. For each $\gamma \in[0,1]$, denote $[\mathscr{T}(u)]^{\gamma}=\left[\mathscr{T}_{*}(u, \gamma), \mathscr{T}^{*}(u, \gamma)\right]$. Thus, a fuzzy mapping $\mathscr{T}$ can be identified by a parametrized triple:

$$
\mathscr{T}(u)=\left\{\left(\mathscr{T}_{*}(u, \gamma), \mathscr{T}^{*}(u, \gamma), \gamma\right): \gamma \in[0,1]\right\} .
$$

Definition 3 (see [40]). Let $L=(m, n)$ and $u \in L$. Then fuzzy mapping $\mathscr{T}:(m, n) \longrightarrow \mathbb{F}_{0}$ is said to be generalized differentiable (in short, G-differentiable) at $u$ if there exists an element $\mathscr{T},(u) \in \mathbb{F}_{0}$.

For all $0<\tau$ sufficiently small, there exist $\mathscr{T}(u+\tau) \sim \mathscr{T}(u), \mathscr{T}(u) \sim \mathscr{T}(u-\tau)$, and the limits (in the metric $D)$.

$$
\begin{aligned}
& \tau \longrightarrow 0^{+} \mathscr{T}(u+\tau) \frac{\sim \mathscr{I}(u)}{\tau}=\lim _{\tau \longrightarrow 0^{+}} \frac{\mathscr{T}(u) \sim \mathscr{T}(u-\tau)}{\tau}=\mathscr{T},(u), \text { or } \\
& \lim _{\tau \longrightarrow 0^{+}} \frac{\mathscr{T}(u) \sim \mathscr{I}(u+\tau)}{-\tau}=\lim _{\tau \longrightarrow 0^{+}} \frac{\mathscr{T}(u-\tau) \tilde{-} \mathscr{T}(u)}{-\tau}=\mathscr{T}^{\prime}(u) \text {, or } \\
& \lim _{\tau \longrightarrow 0^{+}} \frac{\mathscr{T}(u+\tau) \sim \mathscr{I}(u)}{\tau}=\lim _{\tau \longrightarrow 0^{+}} \frac{\mathscr{T}(u-\tau) \tilde{\sim} \mathscr{T}(u)}{-\tau}=\mathscr{T}^{\prime}(u), \text { or } \\
& \lim _{\tau \longrightarrow 0^{+}} \frac{\mathscr{T}(u) \sim \mathscr{T}(u+\tau)}{-\tau}=\lim _{\tau \longrightarrow 0^{+}} \frac{\mathscr{T}(u) \sim \mathscr{T}(u-\tau)}{\tau}=\mathscr{T}^{\prime}(u),
\end{aligned}
$$

where the limits are taken in the metric space $\left(\mathbb{F}_{0}, D\right)$, for $\psi, \phi \in \mathbb{F}_{0}$.

$$
D(\psi, \phi)=\sup _{0 \leq \gamma \leq 1} H\left(\psi \gamma, \phi_{\gamma}\right)
$$

and $H$ denotes the well-known Hausdorff metric on space of intervals.

Definition 4 (see [27]). A fuzzy mapping $\mathscr{T}: K \longrightarrow \mathbb{F}_{0}$ is called convex on the convex set $K$ if 


$$
\mathscr{T}((1-\tau) u+\tau \vartheta) \preccurlyeq(1-\tau) \mathscr{T}(u) \tilde{+} \tau \mathscr{T}(\vartheta), \quad \forall u, \vartheta \in K \tau \in[0,1] .
$$

It is strictly convex fuzzy mapping if strict inequality holds for $\mathscr{T}(u) \neq \mathscr{T}(\mathcal{\vartheta}) . \mathscr{T}: K \longrightarrow \mathbb{F}_{0}$ is said to be concave fuzzy mapping if $-\mathscr{T}$ is convex on $K$. It is strictly concave fuzzy mapping if strict inequality holds for $\mathscr{T}(u) \neq \mathscr{T}(\vartheta)$.

Definition 5 (see [27]). A fuzzy mapping $\mathscr{T}: K \longrightarrow \mathbb{F}_{0}$ is called quasi-convex on the convex set $K$ if

$$
\mathscr{T}((1-\tau) u+\tau \vartheta) \preccurlyeq \max (\mathscr{T}(u), \mathscr{T}(\vartheta)), \quad \forall u, \vartheta \in K, \tau \in[0,1] .
$$

Definition 6 (see [2]). The set $K_{\xi}$ in $\mathbb{R}$ is said to be invex set with respect to arbitrary bifunction $\xi(.,$.$) , if$

$$
u+\tau \xi(\vartheta, u) \in K_{\xi}, \quad \forall u, \vartheta \in K_{\xi}, \tau \in[0,1] .
$$

The invex set $K_{\xi}$ is also called $\xi$-connected set. Note that convex set $\xi(\vartheta, u)=\vartheta-u$ is called an invex set in classical sense, but the converse is not valid. For instance, the set $K_{\xi}=$ $[-7,-2] \cup[2,10]$ is an invex set with respect to nontrivial bifunction $\xi: \mathbb{R} \times \mathbb{R} \longrightarrow \mathbb{R}$ given as

$$
\begin{aligned}
& \xi(\vartheta, u)=\vartheta-u, \quad \vartheta \geq 0, u \geq 0, \\
& \xi(\vartheta, u)=\vartheta-u, \quad 0 \geq \vartheta, 0 \geq u, \\
& \xi(\vartheta, u)=-7-u, \quad \vartheta \geq 0 \geq u, \\
& \xi(\vartheta, u)=2-u, \quad u \geq 0 \geq \vartheta .
\end{aligned}
$$

Definition 7 (see [17]). A fuzzy mapping $\mathscr{T}: K_{\xi} \longrightarrow \mathbb{F}_{0}$ is called preinvex on the invex set $K_{\xi}$ with respect to bifunction $\xi$ if

$$
\mathscr{T}(u+\tau \xi(\vartheta, u)) \preccurlyeq(1-\tau) \mathscr{T}(u) \tilde{+} \tau \mathscr{T}(\vartheta),
$$

$\forall u, \vartheta \in K_{\xi}, \tau \in[0,1]$, where $\xi: K_{\xi} \times K_{\xi} \longrightarrow \mathbb{R}$.

It is strictly preinvex fuzzy mapping if strict inequality holds for $\mathscr{T}(u) \neq \mathscr{T}(\vartheta)$. $\mathscr{T}: K_{\xi} \longrightarrow \mathbb{F}_{0}$ is said to be preconcave fuzzy mapping if $-\mathscr{T}$ is preinvex on $K_{\xi}$. It is strictly preconcave fuzzy mapping if strict inequality holds for $\mathscr{T}(u) \neq \mathscr{T}(\vartheta)$.

Lemma 1. (see [42]). Let $K_{\xi}$ be an invex set with respect to $\xi$ and let $\mathscr{T}: K_{\xi} \longrightarrow \mathbb{F}_{0}$ be a fuzzy mapping parametrized by

$$
\mathscr{T}(u)=\left\{\left(\mathscr{T}_{*}(u, \gamma), \mathscr{T}^{*}(u, \gamma), \gamma\right): \gamma \in[0,1]\right\},
$$

$\forall u \in K_{\xi}$. Then $\mathscr{T}$ is preinvex on $K_{\xi}$ if and only if, for all $\gamma \in[0,1], \mathscr{T}_{*}(u, \gamma)$ and $\mathscr{T}^{*}(u, \gamma)$ are preinvex with respect to $\xi$ on $K_{\xi}$.

Definition 8 (see [17]). A comparable fuzzy mapping $\mathscr{T}: K_{\xi} \longrightarrow \mathbb{F}_{0}$ is called quasi-preinvex on the invex set $K_{\xi}$ with respect to $\xi$ if, $\forall u, \vartheta \in K_{\xi}, \tau \in[0,1]$ such that

$$
\mathscr{T}(u+\tau \xi(\vartheta, u)) \preccurlyeq \max (\mathscr{T}(u), \mathscr{T}(\vartheta)) .
$$

For further study, let $K_{\xi}$ be a nonempty closed invex set in $\mathbb{R}$. Let $\mathscr{T}: K_{\xi} \longrightarrow \mathbb{F}_{0}$ be a fuzzy mapping and let $\xi: K_{\xi} \times$ $K_{\xi} \longrightarrow \mathbb{R}$ be an arbitrary bifunction. Let $\Omega($.) be a nonnegative function, defined as $\Omega: \mathbb{R} \longrightarrow \mathbb{R}^{+}$. We let $\|$.$\| and$ $\langle.,$.$\rangle be the norm and inner product, respectively.$

\section{Strongly Generalized Preinvex Fuzzy Mappings}

In this section, we propose the new concept of nonconvex fuzzy mappings known as strongly generalized preinvex fuzzy mappings. We define some different types of nonconvex fuzzy mappings and investigate some basic properties.

Definition 9. Let $K_{\xi}$ be an invex set and let $\mathscr{T}: K_{\xi} \longrightarrow \mathbb{F}_{0}$ be fuzzy mapping. Then $\mathscr{T}$ is said to be strongly generalized preinvex fuzzy mapping with respect to an arbitrary nonnegative function $\Omega($.$) and bifunction \xi(.,$.$) , if there exists a$ constant $\omega$ such that

$$
\mathscr{T}(u+\tau \xi(\vartheta, u)) \leq(1-\tau) \mathscr{T}(u) \tilde{+} \tau \mathscr{T}(\vartheta) \sim \omega \tau(1-\tau) \Omega(\xi(\vartheta, u)),
$$

$\forall u, \vartheta \in K_{\xi}, \tau \in[0,1] . \xi: K_{\xi} \times K_{\xi} \longrightarrow \mathbb{R}$ and $\Omega: \mathbb{R} \longrightarrow \mathbb{R}^{+}$.

It is strictly strongly generalized preinvex fuzzy mapping if strict inequality holds for $\mathscr{T}(u) \neq \mathscr{T}(\vartheta)$ and $\mathscr{T}: K_{\xi} \longrightarrow \mathbb{F}_{0}$ is said to be strongly generalized preconcave fuzzy mapping if $-\mathscr{T}$ is strongly generalized preinvex on $K_{\xi}$. It is strictly generalized preconcave fuzzy mapping if strict inequality holds for $\mathscr{T}(u) \neq \mathscr{T}(\vartheta)$.

Now we discuss some special cases of strongly generalized preinvex fuzzy mappings.

If $\Omega(\vartheta, u)=\|\xi(\vartheta, u)\|^{\rho}, \quad \rho>0, \quad$ then, $\quad$ for all $u, \vartheta \in K_{\xi}, \tau \in[0,1]$, (4) becomes

$$
\mathscr{T}(u+\tau\|\xi(\vartheta, u)\|) \leq(1-\tau) \mathscr{T}(u) \tilde{+} \tau \mathscr{T}(\vartheta)-\omega \tau(1-\tau) \xi(\vartheta, u)^{\rho},
$$

which is called the higher-ordered strongly preinvex fuzzy mapping. This is itself a very interesting problem to study its applications in pure and applied science like fuzzy optimization. When $\rho=2, \mathscr{T}$ is called strongly preinvex fuzzy mapping with respect to bifunction $\xi$.

If $\omega=0$, then strongly generalized preinvex fuzzy mapping becomes preinvex fuzzy mapping with respect to bifunction $\xi(.,$.$) ; that is,$

$$
\mathscr{T}(u+\tau \xi(\vartheta, u)) \leq(1-\tau) \mathscr{T}(u) \tilde{+} \tau \mathscr{T}(\vartheta), \quad \forall u, \vartheta \in K_{\xi}, \tau \in[0,1] .
$$

If $\Omega(\xi(\vartheta, u))=\|\vartheta-u\|^{\rho}$, with $\xi(\vartheta, u)=\vartheta-u$ and $\rho>0$, then $\forall u, \vartheta \in K_{\xi}, \tau \in[0,1]$, and inequality (4) becomes

$$
\mathscr{T}(u+\tau \xi(\vartheta, u)) \preccurlyeq(1-\tau) \mathscr{T}(u) \tilde{+} \tau \mathscr{T}(\vartheta)-\omega \tau(1-\tau)\|\vartheta-u\|^{\rho},
$$

and this is called the higher-ordered strongly convex fuzzy mapping. When $\rho=2, \mathscr{T}$ is called strongly convex fuzzy mapping. 
If $\omega=0$, then higher-ordered strongly convex fuzzy mapping becomes convex fuzzy mapping; that is,

$\mathscr{T}(u+\tau(\vartheta-u)) \preccurlyeq(1-\tau) \mathscr{T}(u) \tilde{+} \tau \mathscr{T}(\vartheta) . \quad \forall u, \vartheta \in K_{\xi}, \tau \in[0,1]$.

If $\tau=(1 / 2)$, then (22) becomes

$\mathscr{T}\left(\frac{2 u+\xi(\vartheta, u)}{2}\right) \leqslant \frac{\mathscr{T}(u) \widetilde{T} \mathscr{T}(\vartheta)}{2} \simeq \frac{1}{4} \omega \Omega(\xi(\vartheta, u)), \quad \forall u, \vartheta \in K_{\xi}$.

Mapping $\mathscr{T}$ is called the strongly generalized Jensen preinvex (in short, $J$-preinvex) fuzzy mapping.

We also define the strongly generalized affine $J$-preinvex fuzzy mapping.

Definition 10. A mapping $\mathscr{T}: K_{\xi} \longrightarrow \mathbb{F}_{0}$ is said to be strongly generalized affine preinvex fuzzy mapping on $K_{\xi}$ with respect to an arbitrary nonnegative function $\Omega$ and bifunction $\xi$, if, $\forall u, \vartheta \in K_{\xi}, \tau \in[0,1]$, there exists a positive number $\omega$ such that

$$
\mathscr{T}(u+\tau \xi(\vartheta, u))=(1-\tau) \mathscr{T}(u) \tilde{+} \tau \mathscr{T}(\vartheta) \sim \omega \tau(1-\tau) \Omega(\xi(\vartheta, u)),
$$

where $\xi: K_{\xi} \times K_{\xi} \longrightarrow \mathbb{R}$ and $\Omega: \mathbb{R} \longrightarrow \mathbb{R}^{+}$.

If $\tau=(1 / 2)$, then we also say that $\mathscr{T}$ is strongly generalized affine $J$-preinvex fuzzy mapping such that

$$
\mathscr{T}\left(\frac{2 u+\xi(\vartheta, u)}{2}\right)=\frac{\mathscr{T}(u) \tilde{+} \mathscr{T}(\vartheta)}{2} \simeq \frac{1}{4} \omega \Omega(\xi(\vartheta, u)),
$$

for all $u, \vartheta \in K_{\xi}$

Remark 2. The strongly generalized preinvex fuzzy mappings have some very nice properties similar to preinvex fuzzy mapping: (i) If $\mathscr{T}$ is strongly generalized preinvex fuzzy mapping, then $\sigma \mathscr{T}$ is also strongly generalized preinvex for $\sigma \geq 0$

(ii) If $\mathscr{T}$ and $\mathscr{G}$ both are strongly generalized preinvex fuzzy mappings with respect to an arbitrary nonnegative function $\Omega($.$) and bifunction \xi(.,$.$) , then$ $\max (\mathscr{T}(u), \mathscr{G}(u))$ is also strongly generalized preinvex fuzzy mapping with respect to $\Omega$ and $\xi$

Theorem 2. Let $K_{\xi}$ be an invex set with respect to $\xi$ and let $\mathscr{T}: K_{\xi} \longrightarrow \mathbb{F}_{0}$ be a fuzzy mapping parametrized by

$$
\mathscr{T}(u)=\left\{\left(\mathscr{T}_{*}(u, \gamma), \mathscr{T}^{*}(u, \gamma), \gamma\right): \gamma \in[0,1]\right\},
$$

for all $u \in K_{\xi}$, and then $\mathscr{T}$ is strongly generalized preinvex fuzzy mapping on $K_{\xi}$ with modulus $\omega$ if and only if, for all $\gamma \in[0,1], \mathscr{T}_{*}(u, \gamma)$ and $\mathscr{T}^{*}(u, \gamma)$ are strongly generalized preinvex functions with respect to $\Omega, \xi$, and modulus $\omega$.

Proof. Assume that, for each $\gamma \in[0,1], \mathscr{T}_{*}(u, \gamma)$ and $\mathscr{T}^{*}(u, \gamma)$ are strongly generalized preinvex functions with respect to $\Omega, \xi$, and modulus $\omega$ on $K_{\xi}$. Then, from (4), for all $u, \vartheta \in K_{\xi}, \tau \in[0,1]$, we have

$$
\begin{aligned}
\mathscr{T}_{*}(u+\tau \xi(\vartheta, u), \gamma) \leq & (1-\tau) \mathscr{T}_{*}(u, \gamma)+\tau \mathscr{T}_{*}(\vartheta, \gamma) \\
& -\omega \tau(1-\tau) \Omega(\xi(\vartheta, u)), \\
\mathscr{T}^{*}(u+\tau \xi(\vartheta, u), \gamma) \leq & (1-\tau) \mathscr{T}^{*}(u, \gamma)+\tau \mathscr{T}^{*}(\vartheta, \gamma) \\
& -\omega \tau(1-\tau) \Omega(\xi(\vartheta, u)) .
\end{aligned}
$$

Then, by (30), (9), (10), and (11), we obtain

$$
\begin{aligned}
& \mathscr{T}(u+\tau \xi(\vartheta, u))=\left\{\left(\mathscr{T}_{*}(u+\tau \xi(\vartheta, u), \gamma), \mathscr{T}^{*}(u+\tau \xi(\vartheta, u), \gamma), \gamma\right): \gamma \in[0,1]\right\}, \\
& \leqslant\left\{\left((1-\tau) \mathscr{T}_{*}(u, \gamma),(1-\tau) \mathscr{T}^{*}(u, \gamma), \gamma\right): \gamma \in[0,1]\right\} \tilde{+}\left\{\left(\tau \mathscr{T}_{*}(\vartheta, \gamma), \tau \mathscr{T}^{*}(\vartheta, \gamma), \gamma\right): \gamma \in[0,1]\right\} \tilde{\sim} \omega \tau(1-\tau) \Omega(\xi(\vartheta, u)), \\
& =(1-\tau) \mathscr{T}(u) \tilde{+} \tau \mathscr{T}(\vartheta) \sim \omega \tau(1-\tau) \Omega(\xi(\vartheta, u)), \quad \forall u, \vartheta \in K_{\xi}, \tau \in[0,1] .
\end{aligned}
$$

Hence, $\mathscr{T}(u)$ is a strongly generalized preinvex fuzzy $\mathscr{T}(u+\tau \xi(\vartheta, u)) \preccurlyeq(1-\tau) \mathscr{T}(u) \tilde{+} \tau \mathscr{T}(\vartheta) \sim \omega \tau(1-\tau) \Omega(\xi(\vartheta, u))$. mapping on $K_{\xi}$ with modulus $\omega$.

Conversely, let $\mathscr{T}(u)$ be a strongly generalized preinvex fuzzy mapping on $K_{\xi}$ with modulus $\omega$. Then, for all $u, \vartheta \in K_{\xi}$ and $\tau \in[0,1]$, we have

$$
\mathscr{T}(u+\tau \xi(\vartheta, u))=\left\{\left(\mathscr{T}_{*}(u+\tau \xi(\vartheta, u), \gamma), \mathscr{T}^{*}(u+\tau \xi(\vartheta, u), \gamma), \gamma\right): \gamma \in[0,1]\right\}, \quad \forall u, \vartheta \in K_{\xi}, \tau \in[0,1]
$$


From (30), (9), (10), and (11), we obtain

$$
\begin{gathered}
(1-\tau) \mathscr{T}(u) \tilde{+} \tau \mathscr{T}(\vartheta) \\
\sim \Omega\left\{\tau^{p}(1-\tau)+\tau(1-\tau)^{p}\right\} \xi(\vartheta, u)^{p}= \\
\left\{\left((1-\tau) \mathscr{T}_{*}(u, \gamma),(1-\tau) \mathscr{T}^{*}(u, \gamma), \gamma\right): \gamma \in[0,1]\right\} \tilde{+}\left\{\left(\tau \mathscr{T}_{*}(\vartheta, \gamma), \tau \mathscr{T}^{*}(\vartheta, \gamma), \gamma\right): \gamma \in[0,1]\right\} \\
\simeq \omega \tau(1-\tau) \Omega(\xi(\vartheta, u)),
\end{gathered}
$$

for all $u, \vartheta \in K_{\xi}$ and $\tau \in[0,1]$. Then, by strongly generalized preinvexity of $\mathscr{T}(u)$, we have, for all $u, \vartheta \in K_{\xi}$ and $\tau \in[0,1]$ such that

$$
\begin{aligned}
& \mathscr{T}_{*}(u+\tau \xi(\vartheta, u), \gamma) \leq(1-\tau) \mathscr{T}_{*}(u, \gamma)+\tau \mathscr{T}_{*}(\vartheta, \gamma)-\omega \tau(1-\tau) \Omega(\xi(\vartheta, u)), \\
& \mathscr{T}^{*}(u+\tau \xi(\vartheta, u), \gamma) \leq(1-\tau) \mathscr{T}^{*}(u, \gamma)+\tau \mathscr{T}^{*}(\vartheta, \gamma)-\omega \tau(1-\tau) \Omega(\xi(\vartheta, u)), \\
& \quad \Omega(\xi(\vartheta, u))=\|\xi(\vartheta, u)\|^{2} \text { and } \xi(\vartheta, u)=\vartheta-u,
\end{aligned}
$$

for each $\gamma \in[0,1]$. Hence, the result follows.

Note that if $\xi(\vartheta, u)=\vartheta-u$, then Theorem 2 reduces to the following statement.

Let $K_{\xi}$ be a convex set and let $\mathscr{T}: K \longrightarrow \mathbb{F}_{0}$ be a fuzzy mapping parametrized by

$$
\mathscr{T}(u)=\left\{\left(\mathscr{T}_{*}(u, \gamma), \mathscr{T}^{*}(u, \gamma), \gamma\right): \gamma \in[0,1]\right\}, \quad \forall u \in K_{\xi},
$$

and then $F$ is a strongly generalized convex fuzzy mapping on $K_{\xi}$ if and only if, for all $\gamma \in[0,1], \mathscr{T}_{*}(u, \gamma)$ and $\mathscr{T}^{*}(u, \gamma)$ are strongly generalized convex functions on $K_{\xi}$

Example 1. We consider the fuzzy mappings $\mathscr{T}:(0, \infty) \longrightarrow \mathbb{F}_{0}$ defined by

$$
\mathscr{T}(u)(\sigma)= \begin{cases}\frac{\sigma}{u^{2}}, & \sigma \in\left[0, u^{2}\right], \\ \frac{2 u^{2}-\sigma}{u^{2}}, & \sigma \in\left(u^{2}, 2 u^{2}\right], \\ 0, & \text { otherwise. }\end{cases}
$$

Then, for each $\gamma \in[0,1]$, we have $\mathscr{T}_{\gamma}(u)=\left[\gamma u^{2},(2-\gamma) u^{2}\right]$. Since end point functions $\mathscr{T}_{*}(\gamma)$, $\mathscr{T}^{*}(\gamma)$ are strongly generalized preinvex for each $\gamma \in[0,1]$, $\mathscr{T}$ is a strongly generalized preinvex fuzzy mapping with respect to and $0<\omega \leq 1$.

From Example 1, it can be easily seen that, for each $\omega \in(0,1]$, there exists a strongly generalized preinvex fuzzy mapping.

We now established a result for strongly generalized preinvex fuzzy mapping, which shows that the difference of strongly generalized preinvex fuzzy mapping and strongly generalized affine preinvex fuzzy mapping is again a strongly generalized preinvex fuzzy mapping.

Theorem 3. Let fuzzy mapping $\mathscr{J}: K_{\xi} \longrightarrow \mathbb{F}_{0}$ be strongly generalized affine preinvex with respect to $\Omega$ and $\xi$. Then $\mathscr{T}$ is strongly generalized preinvex fuzzy with respect to same $\Omega$ and $\xi$ if and only if $\mathscr{G}=\mathscr{T}^{\sim} \mathscr{J}$ is a preinvex fuzzy mapping.

Proof. The "If" part is obvious. To prove the "only if" part, assume that $\mathscr{J}: K_{\xi} \longrightarrow \mathbb{F}_{0}$ is strongly generalized affine preinvex with respect to nonnegative function $\Omega$ and bifunction $\xi$; then there exists $\omega>0$ such that

$$
\mathscr{J}(u+\tau \xi(\vartheta, u))=(1-\tau) \mathscr{F}(u) \tilde{+} \tau \mathscr{J}(\vartheta) \sim \omega \tau(1-\tau) \Omega(\xi(\vartheta, u)),
$$

and since $\mathscr{T}$ is a strongly generalized preinvex fuzzy mapping, we have

$$
\mathscr{T}(u+\tau \xi(\vartheta, u)) \preccurlyeq(1-\tau) \mathscr{T}(u) \tilde{+} \tau \mathscr{T}(\vartheta) \tilde{-} \omega \tau(1-\tau) \Omega(\xi(\vartheta, u)),
$$

and, from (40) and (41), we have

$$
\begin{aligned}
\mathscr{T}(u+\tau \xi(\vartheta, u)) \sim \mathscr{J}(u+\tau \xi(\vartheta, u)) \preccurlyeq(1-\tau) \mathscr{T}(u) \tilde{+} \tau \mathscr{T}(\vartheta) \sim(1-\tau) \mathscr{J}(u) \sim \tau \mathscr{J}(\vartheta), \\
=(1-\tau)(\mathscr{T}(u) \simeq \mathscr{J}(u)) \tilde{+} \tau(\mathscr{T}(\vartheta) \sim \mathcal{J}(\vartheta)),
\end{aligned}
$$


from which it follows that

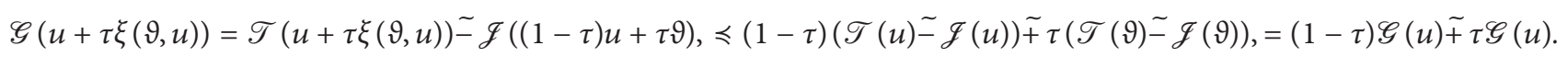

It is shown that $\mathscr{G}=\mathscr{T} \sim$ is a strongly generalized preinvex fuzzy mapping.

Definition 11. A comparable fuzzy mapping $\mathscr{T}: K_{\xi} \longrightarrow \mathbb{F}_{0}$ is said to be strongly generalized quasi-preinvex on $K_{\xi}$ with respect to nonnegative function $\Omega$ and bifunction $\xi$ if, $\forall u, \vartheta \in K_{\xi}, \tau \in[0,1]$, there exists a positive number $\omega$ such that

$$
\mathscr{T}(u+\tau \xi(\vartheta, u)) \preccurlyeq \max (\mathscr{T}(u), \mathscr{T}(\vartheta)) \sim \omega \tau(1-\tau) \Omega(\xi(\vartheta, u)),
$$

where $\xi: K_{\xi} \times K_{\xi} \longrightarrow \mathbb{R}$ and $\Omega: \mathbb{R} \longrightarrow \mathbb{R}^{+}$.
Definition 12. A fuzzy mapping $\mathscr{T}: K_{\xi} \longrightarrow \mathbb{F}_{0}$ is called strongly generalized log-preinvex on $K_{\xi}$ with respect to nonnegative function $\Omega$ and bifunction $\xi$, if, $\forall u, \vartheta \in K_{\xi}, \tau \in[0,1]$, there exists a positive number $\omega$ such that

$$
\mathscr{T}(u+\tau \xi(\vartheta, u)) \preccurlyeq(\mathscr{T}(u))^{1-\tau}(\mathscr{T}(\vartheta))^{\tau} \simeq \omega \tau(1-\tau) \Omega(\xi(\vartheta, u))
$$

where $\mathscr{T}()>.\widetilde{0}, \xi: K_{\xi} \times K_{\xi} \longrightarrow \mathbb{R}$ and $\Omega: \mathbb{R} \longrightarrow \mathbb{R}^{+}$.

$$
\begin{aligned}
\mathscr{T}(u+ & \tau \xi(\vartheta, u)) \preccurlyeq(\mathscr{T}(u))^{1-\tau}(\mathscr{T}(\vartheta))^{\tau}-\omega \tau(1-\widetilde{\tau}) \Omega(\xi(\vartheta, u)), \\
& \preccurlyeq(1-\tau) \mathscr{T}(u) \tilde{+} \tau \mathscr{T}(\vartheta) \sim \omega \tau(1-\tau) \Omega(\xi(\vartheta, u)) \\
& \leqslant \max (\mathscr{T}(u), \mathscr{T}(\vartheta)) \sim \omega \tau(1-\tau) \Omega(\xi(\vartheta, u)) .
\end{aligned}
$$

It can be easily seen that each strongly generalized logpreinvex fuzzy mapping on $K_{\xi}$ is strongly generalized quasipreinvex fuzzy mapping and strongly generalized preinvex fuzzy mapping on $K_{\xi}$ is strongly generalized quasi-preinvex fuzzy mapping, when $\mathscr{T}$ is a comparable fuzzy mapping.

Definition 13. A fuzzy mapping $\mathscr{T}: K_{\xi} \longrightarrow \mathbb{F}_{0}$ is called pseudo-preinvex on $K_{\xi}$ if there exists a strictly positive bifuzzy mapping $b(.,$.$) such that$

$$
\mathscr{T}(\vartheta) \prec \mathscr{T}(u) \Rightarrow \mathscr{T}(u+\tau \xi(\vartheta, u)) \prec \mathscr{T}(u) \tilde{+} \tau(\tau-1) b(u, \vartheta),
$$

for all $u, \vartheta \in K_{\xi}, \tau \in[0,1]$, where $b: K_{\xi} \times K_{\xi} \longrightarrow \mathbb{F}_{0}$.

Theorem 4. Let $\mathscr{T}$ be a strongly generalized preinvex fuzzy mapping on $K_{\xi}$ such that $\mathscr{T}(\vartheta) \prec \mathscr{T}(u)$. Then fuzzy mapping $\mathscr{T}$ is strongly generalized pseudo-preinvex with respect to same nonnegative function $\Omega$ and $\xi$.

Proof. Let $\mathscr{T}(\vartheta) \prec \mathscr{T}(u)$ and let $\mathscr{T}$ be a strongly generalized preinvex fuzzy mapping. Then, for all , $\vartheta \in K_{\xi}, \tau \in[0,1]$, there exists modulus $\omega$ such that
$\mathscr{T}(u+\tau \xi(\vartheta, u)) \preccurlyeq(1-\tau) \mathscr{T}(u) \tilde{+} \tau \mathscr{T}(\vartheta) \tilde{\sim} \omega \tau(1-\tau) \Omega(\xi(\vartheta, u))$,

and, therefore, for every $\gamma \in[0,1]$, we have

$$
\begin{aligned}
\mathscr{T}_{*}(u+\tau \xi(\vartheta, u), \gamma) \leq & (1-\tau) \mathscr{T}_{*}(u, \gamma)+\tau \mathscr{T}_{*}(\vartheta, \gamma) \\
& -\omega \tau(1-\tau) \Omega(\xi(\vartheta, u)), \\
\mathscr{T}^{*}(u+\tau \xi(\vartheta, u), \gamma) \leq & (1-\tau) \mathscr{T}^{*}(u, \gamma)+\tau \mathscr{T}^{*}(\vartheta, \gamma) \\
& -\omega \tau(1-\tau) \Omega(\xi(\vartheta, u)) .
\end{aligned}
$$

From the above equation, we have

$$
\begin{aligned}
\mathscr{T}_{*}(u+\tau \xi(\vartheta, u), \gamma) \leq & \mathscr{T}_{*}(u, \gamma)+\tau\left\{\mathscr{T}_{*}(\vartheta, \gamma)-\mathscr{T}_{*}(u, \gamma)\right\} \\
& -\omega \tau(1-\tau) \Omega(\xi(\vartheta, u)), \\
\mathscr{T}^{*}(u+\tau \xi(\vartheta, u), \gamma) \leq & \mathscr{T}^{*}(u, \gamma)+\tau\left\{\mathscr{T}^{*}(\vartheta, \gamma)-\mathscr{T}^{*}(u, \gamma)\right\} \\
& -\omega \tau(1-\tau) \Omega(\xi(\vartheta, u)) .
\end{aligned}
$$

From (50), we have 


$$
\begin{aligned}
& \mathscr{T}_{*}(u+\tau \xi(\vartheta, u), \gamma)<\mathscr{T}_{*}(u, \gamma)+\tau(\tau-1)\left\{\mathscr{T}_{*}(u, \gamma)-\mathscr{T}_{*}(\vartheta, \gamma)\right\}-\omega \tau(1-\tau) \Omega(\xi(\vartheta, u)), \\
& \mathscr{T}^{*}(u+\tau \xi(\vartheta, u), \gamma)<\mathscr{T}^{*}(u, \gamma)+\tau(\tau-1)\left\{\mathscr{T}^{*}(u, \gamma)-\mathscr{T}^{*}(\vartheta, \gamma)\right\}-\omega \tau(1-\tau) \Omega(\xi(\vartheta, u)), \\
& \mathscr{T}_{*}(u+\tau \xi(\vartheta, u), \gamma)<\mathscr{T}_{*}(u, \gamma)+\tau(\tau-1) b_{*}((u, \vartheta), \gamma)-\omega \tau(1-\tau) \Omega(\xi(\vartheta, u)), \\
& \mathscr{T}^{*}(u+\tau \xi(\vartheta, u), \gamma)<\mathscr{T}^{*}(u, \gamma)+\tau(\tau-1) b^{*}((u, \vartheta), \gamma)-\omega \tau(1-\tau) \Omega(\xi(\vartheta, u)),
\end{aligned}
$$

that is

$$
\mathscr{T}(u+\tau \xi(\vartheta, u))<\mathscr{T}(u) \tilde{+} \tau(\tau-1) b(u, \vartheta) \sim \omega \tau(1-\tau) \Omega(\xi(\vartheta, u)),
$$

where $b(u, 9)=\mathscr{T}(u) \sim \mathscr{T}(9)$. This completes the proof.

\section{G-Differentiable Strongly Generalized Preinvex Fuzzy Mappings}

Throughout this section, we assume that the nonnegative function $\Omega$ is homogenous of degree two and even; that is, $(\rho u)=\rho^{2} \Omega(u), \Omega(-u)=\Omega(u)$, for all $u \in K_{\xi}, \rho \in \mathbb{R}$, unless otherwise specified.

Definition 14. A fuzzy mapping $\mathscr{T}: K_{\xi} \longrightarrow \mathbb{F}_{0}$ is called sharply strongly generalized pseudo-preinvex with respect to $\Omega$ and $\xi$ on $K_{\xi}$ if, $\forall u, \vartheta \in K_{\xi}, \tau \in[0,1]$, there exists $\omega>0$ such that

$$
\left\langle\mathscr{T}^{\prime}(u), \xi(\vartheta, u)\right\rangle \geqslant \widetilde{0} \Rightarrow \mathscr{T}(\vartheta+\tau \xi(\vartheta, u)) \tilde{+} \omega \tau(1-\tau) \Omega(\xi(\vartheta, u)) \preccurlyeq(\vartheta),
$$

where $\mathscr{T}$, is G-differentiable of $\mathscr{T}$ at $u$.

Theorem 5. Let $\mathscr{T}$ be a sharply strongly generalized pseudopreinvex fuzzy mapping on $K_{\xi}$ with $\omega>0$. Then,

$$
\left.\stackrel{\sim}{\sim} \mathscr{T}^{\prime}(\vartheta), \xi(\vartheta, u)\right\rangle \geqslant \omega \Omega(\xi(\vartheta, u)), \quad \forall u, \vartheta \in K_{\xi} .
$$

Proof. Since $\mathscr{T}$ is a sharply strongly generalized pseudopreinvex fuzzy mapping on $K_{\xi}, \forall u, \vartheta \in K_{\xi}, \tau \in[0,1]$, there exists $\omega>0$ such that

$$
\left\langle\mathscr{T}^{\prime}(u), \xi(\vartheta, u)\right\rangle \geqslant \widetilde{0},
$$

which implies that

$$
\mathscr{T}(\vartheta) \succcurlyeq(\vartheta+\tau \xi(\vartheta, u)) \tilde{+} \omega \tau(1-\tau) \Omega(\xi(\vartheta, u)),
$$

and, therefore, for every $\gamma \in[0,1]$, we have

$$
\begin{aligned}
& \mathscr{T}_{*}(\vartheta, \gamma) \leq \mathscr{T}_{*}(\vartheta+\tau \xi(\vartheta, u), \gamma)+\omega \tau(1-\tau) \Omega(\xi(\vartheta, u)), \\
& \mathscr{T}^{*}(\vartheta, \gamma) \leq \mathscr{T}^{*}(\vartheta+\tau \xi(\vartheta, u), \gamma)+\omega \tau(1-\tau) \Omega(\xi(\vartheta, u)),
\end{aligned}
$$

from which we get

$$
\begin{aligned}
& \frac{\mathscr{T}_{*}(\vartheta+\tau \xi(\vartheta, u))-\mathscr{T}_{*}(\vartheta)}{\tau}+\omega(1-\tau) \Omega(\xi(\vartheta, u)) \leq 0, \\
& \frac{\mathscr{T}^{*}(\vartheta+\tau \xi(\vartheta, u))-\mathscr{T}^{*}(\vartheta)}{\tau}+\omega(1-\tau) \Omega(\xi(\vartheta, u)) \leq 0,
\end{aligned}
$$

and, taking limit in the above inequality as $\tau \longrightarrow 0$, we get

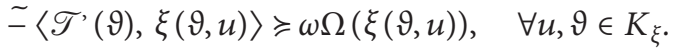

Hence, the result follows.

Definition 15. A G-differentiable fuzzy mapping $\mathscr{T}: K_{\xi} \longrightarrow \mathbb{F}_{0}$ is called strongly generalized invex with respect to $\Omega$ and $\xi$ if, $\forall u, \vartheta \in K_{\xi}$, there exists constant $\omega>0$ such that

$$
\mathscr{T}(\vartheta) \sim \mathscr{T}(u) \geqslant\left\langle\mathscr{T}^{\prime}(u), \xi(\vartheta, u)\right\rangle \tilde{+} \omega \Omega(\xi(\vartheta, u)) .
$$

Definition 16. A G-differentiable fuzzy mapping $\mathscr{T}: K_{\xi} \longrightarrow \mathbb{F}_{0}$ is called strongly generalized pseudo-invex with respect to $\Omega$ and $\xi$ if, $\forall u, \vartheta \in K_{\xi}$, there exists constant $\omega>0$ such that

$$
\left\langle\mathscr{T}^{\prime}(u), \xi(\vartheta, u)\right\rangle \tilde{+} \omega \Omega(\xi(\vartheta, u)) \geqslant \widetilde{0} \Rightarrow \mathscr{T}(\vartheta) \sim \mathscr{T}(u) \geqslant \widetilde{0} .
$$

Definition 17. A G-differentiable fuzzy mapping $\mathscr{T}: K_{\xi} \longrightarrow \mathbb{F}_{0}$ is called strongly generalized quasi-invex with respect to $\Omega$ and $\xi$ if, $\forall u, \vartheta \in K_{\xi}$, there exists constant $\omega>0$ such that

$$
\mathscr{T}(\vartheta) \preccurlyeq(u) \Rightarrow\left\langle\mathscr{T}^{\prime}(u), \xi(\vartheta, u)\right\rangle \tilde{+} \omega \Omega(\xi(\vartheta, u)) \preccurlyeq \widetilde{0} .
$$

Definition 18. A G-differentiable fuzzy mapping $\mathscr{T}: K_{\xi} \longrightarrow \mathbb{F}_{0}$ is called pseudo-invex with respect to $\Omega$ and $\xi$ if, $\forall u, \vartheta \in K_{\xi}$ such that

$$
\left\langle\mathscr{T}^{\prime}(u), \xi(\vartheta, u)\right\rangle \geqslant \widetilde{0} \Rightarrow \mathscr{T}(\vartheta) \sim \mathscr{T}(u)>\widetilde{0} .
$$

Definition 19. A G-differentiable fuzzy mapping $\mathscr{T}: K_{\xi} \longrightarrow \mathbb{F}_{0}$ is called quasi-invex with respect to $\Omega$ and $\xi$ if, $\forall u, \vartheta \in K_{\xi}$ such that

$$
\mathscr{T}(\vartheta) \preccurlyeq(u) \Rightarrow\left\langle\mathscr{T}^{\prime}(u), \xi(\vartheta, u)\right\rangle \preccurlyeq \widetilde{0} .
$$

If $\xi(\vartheta, u)=-\xi(u, \vartheta)$, then definitions reduce to known ones. All Definitions 15-19 may play an important role in fuzzy optimization problem and mathematical programing. 
Theorem 6. Let $\mathscr{T}: K_{\xi} \longrightarrow \mathbb{F}_{0}$ be a G-differentiable strongly generalized preinvex fuzzy mapping. Then $\mathscr{T}$ is a strongly generalized invex fuzzy mapping.
Proof. Let $\mathscr{T}: K_{\xi} \longrightarrow \mathbb{F}_{0}$ be a G-differentiable strongly generalized preinvex fuzzy mapping. Since $\mathscr{T}$ is a strongly generalized preinvex fuzzy mapping, there exists constant $\omega>0$, and, for all $u, \vartheta \in K_{\xi}$ and $\tau \in[0,1]$, we have

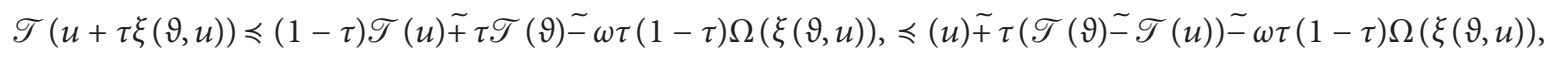

and, therefore, for every $\gamma \in[0,1]$, we have

$$
\begin{aligned}
& \mathscr{T}_{*}(u+\tau \xi(\vartheta, u), \gamma) \leq \mathscr{T}_{*}(u, \gamma)+\tau\left(\mathscr{T}_{*}(\vartheta, \gamma)-\mathscr{T}_{*}(u, \gamma)\right)-\omega \tau(1-\tau) \Omega(\xi(\vartheta, u)), \\
& \mathscr{T}^{*}(u+\tau \xi(\vartheta, u), \gamma) \leq \mathscr{T}^{*}(u, \gamma)+\tau\left(\mathscr{T}^{*}(\vartheta, \gamma)-\mathscr{T}^{*}(u, \gamma)\right)-\omega \tau(1-\tau) \Omega(\xi(\vartheta, u)),
\end{aligned}
$$

which implies that

$$
\begin{array}{r}
\tau\left(\mathscr{T}_{*}(\vartheta, \gamma)-\mathscr{T}_{*}(u, \gamma)\right) \geq \mathscr{T}_{*}(u+\tau \xi(\vartheta, u), \gamma)-\mathscr{T}_{*}(u, \gamma)+\omega \tau(1-\tau) \Omega(\xi(\vartheta, u)), \\
\tau\left(\mathscr{T}^{*}(\vartheta, \gamma)-\mathscr{T}^{*}(u, \gamma)\right) \geq \mathscr{T}^{*}(u+\tau \xi(\vartheta, u), \gamma)-\mathscr{T}^{*}(u, \gamma)+\omega \tau(1-\tau) \Omega(\xi(\vartheta, u)), \\
\mathscr{T}_{*}(\vartheta, \gamma)-\mathscr{T}_{*}(u, \gamma) \geq \frac{\mathscr{T}_{*}(u+\tau \xi(\vartheta, u), \gamma)-\mathscr{T}_{*}(u, \gamma)}{\tau}+\omega(1-\tau) \Omega(\xi(\vartheta, u)), \\
\mathscr{T}^{*}(\vartheta, \gamma)-\mathscr{T}^{*}(u, \gamma) \geq \frac{\mathscr{T}^{*}(u+\tau \xi(\vartheta, u), \gamma)-\mathscr{T}^{*}(u, \gamma)}{\tau}+\omega(1-\tau) \Omega(\xi(\vartheta, u)) .
\end{array}
$$

Taking limit in the above inequality as $\tau \longrightarrow 0$, we have $\mathscr{T}_{*}(\vartheta, \gamma)-\mathscr{T}_{*}(u, \gamma) \geq\left\langle\mathscr{T}_{*}^{\prime}(u, \gamma), \xi(\vartheta, u)\right\rangle+\omega \Omega(\xi(\vartheta, u))$, $\mathscr{T}^{*}(\vartheta, \gamma)-\mathscr{T}^{*}(u, \gamma) \geq\left\langle\mathscr{T}^{*}(u, \gamma), \xi(\vartheta, u)\right\rangle+\omega \Omega(\xi(\vartheta, u))$.

From that, we have

$$
\mathscr{T}(\vartheta) \sim \mathscr{T}(u) \geqslant\left\langle\mathscr{T}^{\prime}(u), \xi(\vartheta, u)\right\rangle \tilde{+} \omega \Omega(\xi(\vartheta, u)),
$$

and when $\omega=0$, Theorem 6 reduces to the following result.

Theorem 7. Let $\mathscr{T}: K_{\xi} \longrightarrow \mathbb{F}_{0}$ be a G-differentiable preinvex fuzzy mapping. Then, $\mathscr{T}$ is an invex fuzzy mapping.
To prove conversion of Theorem 6, we need the following assumption regarding the function $\xi$, which plays an important role in G-differentiation of the main results.

Condition $C$ is as follows [33]:

$$
\begin{aligned}
& \xi(\vartheta, u+\tau \xi(\vartheta, u))=(1-\tau) \xi(\vartheta, u), \\
& \xi(u, u+\tau \xi(\vartheta, u))=-\tau \xi(\vartheta, u) .
\end{aligned}
$$

The following result finds the conversion of Theorem 6 .

Theorem 8. Let $\mathscr{T}$ be a G-differentiable fuzzy mapping on $K_{\xi}$ and Condition $C$ holds. Let the function $\Omega$ be homogenous of degree 2 and even. Then the following are equivalent:

(a) $\mathscr{T}$ is a strongly generalized preinvex fuzzy mapping:

$$
\begin{gathered}
(b) \mathscr{T}(\vartheta) \sim \mathcal{T}(u) \geqslant\left\langle\mathscr{T}^{\prime}(u), \xi(\vartheta, u)\right\rangle \tilde{+} \omega \Omega(\xi(\vartheta, u)), \\
(c)\left\langle\mathscr{T}^{\prime}(u), \xi(\vartheta, u)\right\rangle \tilde{+}\left\langle\mathscr{T}^{\prime}(\vartheta), \xi(u, \vartheta)\right\rangle \preccurlyeq \tilde{-} \omega(\Omega(\xi(\vartheta, u)) \tilde{+} \Omega(\xi(u, \vartheta))), \quad \forall u, \vartheta \in K_{\xi} .
\end{gathered}
$$

Proof. (a) implies (b).

The demonstration is analogous to the demonstration of Theorem 6. (b) implies (c). Assume that (71) holds. Then, $\mathscr{T}(\vartheta) \sim \mathscr{T}(u) \geqslant\left\langle\mathscr{T}^{\prime}(u), \xi(\vartheta, u)\right\rangle \tilde{+} \omega \Omega(\xi(\vartheta, u))$. 
Then, for every $\gamma \in[0,1]$, we have

$\mathscr{T}_{*}(\vartheta, \gamma)-\mathscr{T}_{*}(u, \gamma) \geq\left\langle\mathscr{T}_{*}^{\prime}(u, \gamma), \xi(\vartheta, u)\right\rangle+\omega \Omega(\xi(\vartheta, u))$,

$\mathscr{T}^{*}(\vartheta, \gamma)-\mathscr{T}^{*}(u, \gamma) \geq\left\langle\mathscr{T}_{*}^{\prime}(u, \gamma), \xi(\vartheta, u)\right\rangle+\omega \Omega(\xi(\vartheta, u))$.

(74)

$$
\begin{aligned}
& \mathscr{T}_{*}(u, \gamma)-\mathscr{T}_{*}(\vartheta, \gamma) \geq\left\langle\mathscr{T}_{*}^{\prime}(\vartheta, \gamma), \xi(u, \vartheta)\right\rangle+\omega \Omega(\xi(u, \vartheta)), \\
& \mathscr{T}^{*}(u, \gamma)-\mathscr{T}^{*}(\vartheta, \gamma) \geq\left\langle\mathscr{T}^{*}(\vartheta, \gamma), \xi(u, \vartheta)\right\rangle+\omega \Omega(\xi(u, \vartheta)) .
\end{aligned}
$$

Adding (74) and (75), we have

Replacing $\vartheta$ by $u$ and $u$ by $\vartheta$ in (21), we have

$$
\begin{gathered}
\left\langle\mathscr{T}_{*}^{\prime}(u, \gamma), \xi(\vartheta, u)\right\rangle+\left\langle\mathscr{T}_{*}^{\prime}(\vartheta, \gamma), \xi(u, \vartheta)\right\rangle \leq-\omega(\Omega(\xi(\vartheta, u))+\Omega(\xi(u, \vartheta))), \\
\left\langle\mathscr{T}^{*}(u, \gamma), \xi(\vartheta, u)\right\rangle+\left\langle\mathscr{T}^{*,}(\vartheta, \gamma), \xi(u, \vartheta)\right\rangle \leq-\omega(\Omega(\xi(\vartheta, u))+\Omega(\xi(u, \vartheta))),
\end{gathered}
$$

that is

$\left\langle\mathscr{T}^{\prime}(u), \xi(\vartheta, u)\right\rangle \tilde{+}\left\langle\mathscr{T}^{\prime}(\vartheta), \xi(u, \vartheta)\right\rangle \preccurlyeq \widetilde{\sim} \omega(\Omega(\xi(\vartheta, u)) \tilde{+} \Omega(\xi(u, \vartheta)))$,

and (c) implies (b). Assume that (72) holds. Then,

$\left\langle\mathscr{T}^{\prime}(\vartheta), \xi(u, \vartheta)\right\rangle \preccurlyeq \widetilde{\sim}\left\langle\mathscr{T}^{\prime}(u), \xi(\vartheta, u)\right\rangle \sim \omega(\Omega(\xi(\vartheta, u)) \tilde{+} \Omega(\xi(u, \vartheta)))$.

(78)

$$
\begin{aligned}
\left\langle\mathscr{T}_{*}^{\prime}(\vartheta, \gamma), \xi(u, \vartheta)\right\rangle \leq & -\left\langle\mathscr{T}_{*}^{\prime}(u, \gamma), \xi(\vartheta, u)\right\rangle \\
& -\omega(\Omega(\xi(\vartheta, u))+\Omega(\xi(u, \vartheta))), \\
\left\langle\mathscr{T}^{*,}(\vartheta, \gamma), \xi(u, \vartheta)\right\rangle \leq & -\left\langle\mathscr{T}^{*,}(u, \gamma), \xi(\vartheta, u)\right\rangle \\
& -\omega(\Omega(\xi(\vartheta, u))+\Omega(\xi(u, \vartheta))) .
\end{aligned}
$$

Since $K_{\xi}$ is an invex set, we have $\vartheta_{\tau}=u+\tau \xi(\vartheta, u) \in K_{\xi}$ for all $u, \vartheta \in K_{\xi}$ and $\tau \in[0,1]$. Taking $\vartheta=\vartheta_{\tau}$ in (23), we get

Therefore, for every $\gamma \in[0,1]$, we have

$$
\begin{array}{r}
\left\langle\mathscr{T}_{*}^{\prime}(u+\tau \xi(\vartheta, u), \gamma), \xi(u, u+\tau \xi(\vartheta, u))\right\rangle \leq-\left\langle\mathscr{T}_{*}^{\prime}(u, \gamma), \xi(u+\tau \xi(\vartheta, u))\right\rangle \\
-\omega(\Omega(u+\tau \xi(\vartheta, u), u)+\Omega(\xi(u, u+\tau \xi(\vartheta, u)))), \\
\left\langle\mathscr{T}^{*,}(u+\tau \xi(\vartheta, u), \gamma), \xi(u, u+\tau \xi(\vartheta, u))\right\rangle\left\langle\mathscr{T}^{*}(u, \gamma), \xi(u+\tau \xi(\vartheta, u))\right\rangle \\
-\omega(\Omega(u+\tau \xi(\vartheta, u), u)+\Omega(\xi(u, u+\tau \xi(\vartheta, u)))) .
\end{array}
$$

By using Condition $\mathrm{C}$ and the fact that $\Omega$ is homogenous of degree 2 and even, we have

Let

$$
\begin{aligned}
& H_{*}(\tau)=\mathscr{T}_{*}(u+\tau \xi(\vartheta, u), \gamma), \\
& H^{*}(\tau)=\mathscr{T}^{*}(u+\tau \xi(\vartheta, u), \gamma),
\end{aligned}
$$

and, taking G-derivative with respect to $\tau$, we get from which, using (81), we have 


$$
\begin{aligned}
& H_{*}^{\prime}(\tau) \geq\left\langle\mathscr{T}_{*}^{\prime}(u, \gamma), \xi(\vartheta, u)\right\rangle+2 \omega \tau \Omega(\xi(\vartheta, u)), \\
& H^{*,}(\tau) \geq\left\langle\mathscr{T}^{*,}(u, \gamma), \xi(\vartheta, u)\right\rangle+2 \omega \tau \Omega(\xi(\vartheta, u)) .
\end{aligned}
$$

Integrating (84) over $[0,1]$ with respect to $\tau$, we get

$$
\begin{array}{r}
H_{*}(1)-H_{*}(0) \geq\left\langle\mathscr{T}_{*}^{*}(u, \gamma), \xi(\vartheta, u)\right\rangle+\omega \Omega(\xi(\vartheta, u)), \\
H^{*}(1)-H^{*}(0) \geq\left\langle\mathscr{T}^{*,}(u, \gamma), \xi(\vartheta, u)\right\rangle+\omega \Omega(\xi(\vartheta, u)) . \\
\mathscr{T}_{*}(u+\xi(\vartheta, u), \gamma)-\left\langle\mathscr{T}_{*}(u, \gamma) \geq \mathscr{T}_{*}^{\prime}(u, \gamma)\right\rangle, \xi(\vartheta, u)+\omega \Omega(\xi(\vartheta, u)), \\
\mathscr{T}^{*}(u+\xi(\vartheta, u), \gamma)-\left\langle\mathscr{T}^{*}(u, \gamma) \geq \mathscr{T}^{*,}(u, \gamma)\right\rangle, \xi(\vartheta, u)+\omega \Omega(\xi(\vartheta, u)) .
\end{array}
$$

Since $\mathscr{T}$ is a strongly generalized preinvex fuzzy mapping, for $\tau=1$, we have

$$
\begin{aligned}
& \mathscr{T}_{*}(\vartheta, \gamma)-\mathscr{T}_{*}(u, \gamma) \geq\left\langle\mathscr{T}_{*}^{*}(u, \gamma), \xi(\vartheta, u)\right\rangle+\omega \Omega(\xi(\vartheta, u)), \\
& \mathscr{T}^{*}(\vartheta, \gamma)-\mathscr{T}^{*}(u, \gamma) \geq\left\langle\mathscr{T}^{*}(u, \gamma), \xi(\vartheta, u)\right\rangle+\omega \Omega(\xi(\vartheta, u)) .
\end{aligned}
$$

From that, we have

$$
\mathscr{T}(\vartheta) \sim \mathscr{T}(u) \geqslant\left\langle\mathscr{T}^{\prime}(u), \xi(\vartheta, u)\right\rangle \tilde{+} \omega \Omega(\xi(\vartheta, u)) .
$$

(b) implies (a). Assume that (71) holds. Since $K_{\xi}$ is an invex set, we have $\vartheta_{\tau}=u+\tau \xi(\vartheta, u) \in K_{\xi}$ for all $u, \vartheta \in K_{\xi}$ and $\tau \in[0,1]$. Taking $\vartheta=\vartheta_{\tau}$ in (19) for each $\gamma \in[0,1]$, we get

$$
\begin{array}{r}
\mathscr{T}_{*}\left(\vartheta_{\tau}, \gamma\right)-\mathscr{T}_{*}(u, \gamma) \geq\left\langle\mathscr{T}_{*}(u, \gamma), \xi\left(\vartheta_{\tau}, u\right)\right\rangle+\omega \Omega\left(\xi\left(\vartheta_{\tau}, u\right)\right), \\
\mathscr{T}^{*}\left(\vartheta_{\tau}\right)-\mathscr{T}^{*}(u, \gamma) \geq\left\langle\mathscr{T}^{*}(u, \gamma), \xi\left(\vartheta_{\tau}, u\right)\right\rangle+\omega \Omega\left(\xi\left(\vartheta_{\tau}, u\right)\right) .
\end{array}
$$

Using Condition $\mathrm{C}$, we have

$$
\begin{aligned}
\mathscr{T}_{*}\left(\vartheta_{\tau}, \gamma\right)-\mathscr{T}_{*}(u, \gamma) \geq & (1-\tau)\left\langle\mathscr{T}_{*}^{\prime}(u, \gamma), \xi(\vartheta, u)\right\rangle \\
& +\omega(1-\tau)^{2} \Omega(\xi(\vartheta, u)), \\
\mathscr{T}^{*}\left(\vartheta_{\tau}, \gamma\right)-\mathscr{T}^{*}(u, \gamma) \geq & (1-\tau)\left\langle\mathscr{T}^{*}(u, \gamma), \xi(\vartheta, u)\right\rangle \\
& +\omega(1-\tau)^{2} \Omega(\xi(\vartheta, u)) .
\end{aligned}
$$

In a similar way, we obtain

$$
\begin{aligned}
& \mathscr{T}_{*}(u, \gamma)-\mathscr{T}_{*}\left(\vartheta_{\tau}, \gamma\right) \geq-\tau\left\langle\mathscr{T}_{*}(u, \gamma), \xi(\vartheta, u)\right\rangle+\omega \tau^{2} \Omega(\xi(\vartheta, u)), \\
& \mathscr{T}^{*}(u, \gamma)-\mathscr{T}^{*}\left(\vartheta_{\tau}, \gamma\right) \geq-\tau\left\langle\mathscr{T}^{* *}(u, \gamma), \xi(\vartheta, u)\right\rangle+\omega \tau^{2} \Omega(\xi(\mathcal{\vartheta}, u)) .
\end{aligned}
$$

Multiplying (89) by $\tau$ and (90) by $(1-\tau)$ and adding the resultant, we have

$$
\begin{aligned}
& \mathscr{T}_{*}\left(\vartheta_{\tau}, \gamma\right) \leq(1-\tau) \mathscr{T}_{*}(u, \gamma)+\tau \mathscr{T}_{*}(\vartheta, \gamma)-\Omega \omega \tau(1-\tau) \Omega(\xi(\vartheta, u)), \\
& \mathscr{T}^{*}\left(\vartheta_{\tau}, \gamma\right) \leq(1-\tau) \mathscr{T}^{*}(u, \gamma)+\tau \mathscr{T}^{*}(\vartheta, \gamma)-\omega \tau(1-\tau) \Omega(\xi(\vartheta, u)),
\end{aligned}
$$

that is

$$
\mathscr{T}(u+\tau \xi(\vartheta, u)) \preccurlyeq(1-\tau) \mathscr{T}(u) \tilde{+} \tau \mathscr{T}(\vartheta) \tilde{\sim} \omega \tau(1-\tau) \Omega(\xi(\vartheta, u)) .
$$

Hence, $\mathscr{T}$ is a strongly generalized preinvex fuzzy mapping with respect to $\Omega$ and $\xi$.
Theorems 6 and 8 enable us to define the following new definitions.

Definition 20. A G-differentiable fuzzy mapping $\mathscr{T}: K_{\xi} \longrightarrow \mathbb{F}_{0}$ is said to be the following:

(i) Strongly generalized monotone fuzzy operator with respect to $\Omega$ and $\xi$ if and only if, $\forall u, \vartheta \in K_{\xi}$, there exists constant $\omega>0$ such that

$\left\langle\mathscr{T}^{\prime}(u), \xi(\vartheta, u)\right\rangle \tilde{+}\left\langle\mathscr{T}^{\prime}(\vartheta), \xi(u, \vartheta)\right\rangle \leqslant \tilde{-}(\Omega(\xi(\vartheta, u)) \tilde{+} \Omega(\xi(u, \vartheta)))$.

(ii) Monotone fuzzy operator with respect to bifunction $\xi$ if and only if, $\forall u, \vartheta \in K_{\xi}$,

$$
\left\langle\mathscr{T}^{\prime}(u), \xi(\vartheta, u)\right\rangle \tilde{+}\left\langle\mathscr{T}^{\prime}(\vartheta), \xi(u, \vartheta)\right\rangle \leqslant \widetilde{0} .
$$

(iii) Strongly generalized pseudomonotone fuzzy operator with respect to $\Omega$ and $\xi$ if and only if, $\forall u, \vartheta \in K_{\xi}$, there exists constant $\omega>0$ such that

$$
\left\langle\mathscr{T}^{\prime}(u), \xi(\vartheta, u)\right\rangle \widetilde{+} \omega \Omega(\xi(\vartheta, u)) \geqslant \widetilde{0} \Rightarrow \widetilde{\langle}\left\langle\mathscr{T}^{\prime}(\mathcal{Y}), \xi(u, \mathcal{Y})\right\rangle \geqslant \widetilde{0} .
$$

(iv) Strictly monotone fuzzy operator with respect to bifunction $\xi$ if and only if

$$
\left\langle\mathscr{T}^{\prime}(u), \xi(\vartheta, u)\right\rangle \tilde{+}\left\langle\mathscr{T}^{\prime}(\vartheta), \xi(u, \vartheta)\right\rangle\left\langle\widetilde{0}, \quad \forall u, \vartheta \in K_{\xi} .\right.
$$

(v) Pseudomonotone fuzzy operator with respect to bifunction $\xi$ if and only if

$$
\left\langle\mathscr{T}^{\prime}(u), \xi(\vartheta, u)\right\rangle \geqslant \widetilde{0} \Rightarrow\left\langle\mathscr{T}^{\prime}(\vartheta), \xi(u, \vartheta)\right\rangle \leqslant \widetilde{0}, \quad \forall u, \vartheta \in K_{\xi} .
$$

(vi) Quasi-monotone fuzzy operator with respect to bifunction $\xi$ if and only if

$$
\left\langle\mathscr{T}^{\prime}(u), \xi(\vartheta, u)\right\rangle>\widetilde{0} \Rightarrow\left\langle\mathscr{T}^{\prime}(\vartheta), \xi(u, \vartheta)\right\rangle \leqslant \widetilde{0}, \quad \forall u, \vartheta \in K_{\xi} .
$$

(vii) Strictly pseudomonotone fuzzy operator with respect to bifunction $\xi$ if and only if 


$$
\left\langle\mathscr{T}^{\prime}(u), \xi(\vartheta, u)\right\rangle \geqslant \widetilde{0} \Rightarrow\left\langle\mathscr{T}^{\prime}(\vartheta), \xi(u, \vartheta)\right\rangle<\widetilde{0}, \quad \forall u, \vartheta \in K_{\xi} .
$$

If $\xi(\vartheta, u)=-\xi(u, \vartheta)$, then Definition 20 reduces to a new one.

As a special case of Theorem 8 , we have the following.

Theorem 9. Let $\mathscr{T}$ be a G-differentiable fuzzy mapping on $K_{\xi}$ and Condition $C$ holds. If the function $\Omega$ is homogenous of degree 2 and even, then $\mathscr{T}$ is a strongly generalized $\xi$-invex fuzzy mapping if and only if $\mathscr{T}$, is a strongly generalized $\xi$-monotone fuzzy operator.

Proof. The proof is similar to that of Theorem 8 .

Theorem 10. Let G-differential $\mathscr{T}$, of fuzzy mapping $\mathscr{T}$ on $K_{\xi}$ be a strongly generalized pseudomonotone fuzzy operator and Condition C holds. Let the function $\Omega$ be homogenous of degree 2 and even. Then, $\mathscr{T}$ is a strongly generalized pseudoinvex fuzzy mapping.

Proof. Let $\mathscr{T}$, be fuzzy strongly generalized pseudomonotone. Then, for all $u, \vartheta \in K_{\xi}$, we have

$$
\left\langle\mathscr{T}^{\prime}(u), \xi(\vartheta, u)\right\rangle \tilde{+} \omega \Omega(\xi(\vartheta, u)) \geqslant \widetilde{0} \Rightarrow \tilde{-}\left\langle\mathscr{T}^{\prime}(\vartheta), \xi(u, \vartheta)\right\rangle \geqslant \widetilde{0} .
$$

Therefore, for every $\gamma \in[0,1]$, we have

$$
\begin{aligned}
& -\left\langle\mathscr{T}_{*}^{*}(\vartheta, \gamma), \xi(u, \vartheta)\right\rangle \geq 0, \\
& -\left\langle\mathscr{T}^{*}(\vartheta, \gamma), \xi(u, \vartheta)\right\rangle \geq 0 .
\end{aligned}
$$

Since $K_{\xi}$ is an invex set, we have $\vartheta_{\tau}=u+\tau \xi(\vartheta, u) \in K_{\xi}$ for all $u, \vartheta \in K_{\xi}$ and $\tau \in[0,1]$. Taking $\vartheta=\vartheta_{\tau}$ in $(101)$, we get

$$
\begin{aligned}
& -\left\langle\mathscr{T}^{\prime}(u+\tau \xi(\vartheta, u), \gamma), \xi(u, u+\tau \xi(\vartheta, u))\right\rangle \geq 0, \\
& -\left\langle\mathscr{T}^{*},(u+\tau \xi(\vartheta, u), \gamma), \xi(u, u+\tau \xi(\vartheta, u))\right\rangle \geq 0 .
\end{aligned}
$$

By using Condition C, we have

$$
\begin{aligned}
& \left\langle\mathscr{T}^{\prime}(u+\tau \xi(\vartheta, u), \gamma), \xi(\vartheta, u)\right\rangle \geq 0, \\
& \left\langle\mathscr{T}^{*}(u+\tau \xi(\vartheta, u), \gamma), \xi(\vartheta, u)\right\rangle \geq 0 .
\end{aligned}
$$

Assume that

$$
\begin{aligned}
& H_{*}(\tau)=\mathscr{T}_{*}(u+\tau \xi(\vartheta, u), \gamma), \\
& H^{*}(\tau)=\mathscr{T}^{*}(u+\tau \xi(\vartheta, u), \gamma),
\end{aligned}
$$

and, taking G-derivative with respect to $\tau$, using (103), we have

$$
\begin{aligned}
& H_{*}^{\prime}(\tau)=\left\langle\mathscr{T}_{*}^{\prime}(u+\tau \xi(\vartheta, u), \gamma), \xi(\vartheta, u)\right\rangle \geq 0, \\
& H^{*,}(\tau)=\left\langle\mathscr{T}^{*}(u+\tau \xi(\vartheta, u), \gamma), \xi(\vartheta, u)\right\rangle \geq 0 .
\end{aligned}
$$

Integrating $(105)$ over $[0,1]$ with respect to $\tau$, we get

$$
\begin{aligned}
& H_{*}(1)-H_{*}(0) \geq 0, \\
& H^{*}(1)-H^{*}(0) \geq 0,
\end{aligned}
$$

which implies that

$$
\begin{aligned}
& \mathscr{T}_{*}(u+\xi(\vartheta, u), \gamma)-\mathscr{T}_{*}(u, \gamma) \geq 0, \\
& \mathscr{T}^{*}(u+\xi(\vartheta, u), \gamma)-\mathscr{T}^{*}(u, \gamma) \geq 0 .
\end{aligned}
$$

From condition (i), we have

$$
\begin{aligned}
& \mathscr{T}_{*}(\vartheta, \gamma)-\mathscr{T}_{*}(u, \gamma) \geq 0, \\
& \mathscr{T}^{*}(\vartheta, \gamma)-\mathscr{T}^{*}(u, \gamma) \geq 0,
\end{aligned}
$$

that is

$$
\mathscr{T}(\vartheta) \sim \mathscr{T}(u) \geqslant \widetilde{0}, \quad \forall \vartheta \in K_{\xi} .
$$

Hence, $\mathscr{T}(u)$ is a strongly generalized pseudo-invex fuzzy mapping.

Theorem 11. Let G-differential $\mathscr{T}$ ' of fuzzy mapping $\mathscr{T}$ on $K_{\xi}$ be a pseudomonotone fuzzy operator and Condition $C$ holds. Then $\mathscr{T}$ is a pseudo-invex fuzzy mapping.

Theorem 12. Let G-differential $\mathscr{T}$ ' of fuzzy mapping $\mathscr{T}$ on $K_{\xi}$ be a quasi-monotone fuzzy operator and Condition $C$ holds. Then $\mathscr{T}$ is a quasi-invex fuzzy mapping.

We now discuss the fuzzy optimality condition for G-differentiable strongly generalized preinvex fuzzy mappings, which is the main motivation of our results.

\section{Strongly Generalized Fuzzy Mixed Variational-Like Inequalities}

A well-known fact in mathematical programing is that variational inequality problem has a close relationship with the optimization problem. Similarly, the fuzzy variational inequality problem also has a close relationship with the fuzzy optimization problem.

Consider the unconstrained fuzzy optimization problem

$$
\min _{u \in K_{\xi}} \mathscr{T}(u),
$$

where $K_{\xi}$ is a subset of $\mathbb{R}$ and $\mathscr{T}: K_{\xi} \longrightarrow \mathbb{F}_{0}$ is a fuzzy mapping.

A point $u \in K_{\xi}$ is called a feasible point. If $u \in K_{\xi}$ and no $\vartheta \in K_{\xi}, \mathscr{T}(u)<\mathscr{T}(\vartheta)$, then $u$ is called an optimal solution, a global optimal solution, or simply a solution to the fuzzy optimization problem.

Theorem 13. Let $\mathscr{T}$ be a G-differentiable strongly generalized preinvex fuzzy mapping modulus $\omega>0$. If $u \in K_{\xi}$ is the minimum of the mapping $\mathscr{T}$, then

$$
\mathscr{T}(\vartheta) \simeq \mathscr{T}(u) \geqslant \omega \Omega(\xi(\vartheta, u)), \quad \forall u, \vartheta \in K_{\xi}
$$

Proof. Let $u \in K_{\xi}$ be a minimum of $\mathscr{T}$. Then, $\forall \vartheta \in K_{\xi}$, we have

$$
\mathscr{T}(u) \preccurlyeq(9) .
$$

Therefore, for every $\gamma \in[0,1]$, we have 


$$
\begin{aligned}
& \mathscr{T}_{*}(u, \gamma) \leq \mathscr{T}_{*}(\vartheta, \gamma), \\
& \mathscr{T}^{*}(u, \gamma) \leq \mathscr{T}^{*}(\vartheta, \gamma) .
\end{aligned}
$$

Since $K_{\xi}$ is an invex set, for all $u, \vartheta \in K_{\xi}, \tau \in[0,1]$, $\vartheta_{\tau}=u+\tau \xi(\vartheta, u) \in K_{\xi}$. Taking $\vartheta=\vartheta_{\tau}$ in (113), we get

$$
\begin{gathered}
0 \leq \frac{\mathscr{T}_{*}(u+\tau \xi(\vartheta, u), \gamma)-\mathscr{T}_{*}(u, \gamma)}{\tau}, \\
0 \leq \frac{\mathscr{T}^{*}(u+\tau \xi(\vartheta, u), \gamma)-\mathscr{T}^{*}(u, \gamma)}{\tau} .
\end{gathered}
$$

Taking limit in the above inequality as $\tau \longrightarrow 0$, we get

$$
\begin{aligned}
& 0 \leq\left\langle\mathscr{T}_{*}^{\prime}(u, \gamma), \xi(\vartheta, u)\right\rangle, \\
& 0 \leq\left\langle\mathscr{T}^{*},(u, \gamma), \xi(\vartheta, u)\right\rangle, \\
& \tilde{0} \preccurlyeq\left\langle\mathscr{T}^{\prime}(u), \xi(\vartheta, u)\right\rangle,
\end{aligned}
$$

and since $\mathscr{T}: K_{\xi} \longrightarrow \mathbb{F}_{0}$ is a G-differentiable strongly generalized preinvex fuzzy mapping,

$$
\begin{aligned}
\mathscr{T}_{*}(u+\tau \xi(\vartheta, u), \gamma) \leq & (1-\tau) \mathscr{T}_{*}(u, \gamma)+\tau \mathscr{T}_{*}(\vartheta, \gamma) \\
& -\omega \tau(1-\tau) \Omega(\xi(\vartheta, u)), \\
\mathscr{T}^{*}(u+\tau \xi(\vartheta, u), \gamma) \leq & (1-\tau) \mathscr{T}^{*}(u, \gamma)+\tau \mathscr{T}^{*}(\vartheta, \gamma) \\
& -\omega \tau(1-\tau) \Omega(\xi(\vartheta, u)), \\
\mathscr{T}_{*}(\vartheta, \gamma)-\mathscr{T}_{*}(u, \gamma) \geq & \frac{\mathscr{T}_{*}(u+\tau \xi(\vartheta, u), \gamma)-\mathscr{T}_{*}(u, \gamma)}{\tau} \\
& +\omega(1-\tau) \Omega(\xi(\vartheta, u)), \\
\mathscr{T}^{*}(\vartheta, \gamma)-\mathscr{T}^{*}(u, \gamma) \geq & \frac{\mathscr{T}^{*}(u+\tau \xi(\vartheta, u), \gamma)-\mathscr{T}^{*}(u, \gamma)}{\tau} \\
& +\omega(1-\tau) \Omega(\xi(\vartheta, u)),
\end{aligned}
$$

and, again taking limit in the above inequality as $\tau \longrightarrow 0$, we get

$$
\begin{aligned}
& \mathscr{T}_{*}(\vartheta, \gamma)-\mathscr{T}_{*}(u, \gamma) \geq\left\langle\mathscr{T}_{*}^{\prime}(u, \gamma), \xi(\vartheta, u)\right\rangle+\omega \Omega(\xi(\vartheta, u)), \\
& \mathscr{T}^{*}(\vartheta, \gamma)-\mathscr{T}^{*}(u, \gamma) \geq\left\langle\mathscr{T}^{*}(u, \gamma), \xi(\vartheta, u)\right\rangle+\omega \Omega(\xi(\vartheta, u)),
\end{aligned}
$$

from which, using (115), we have

$$
\begin{aligned}
& \mathscr{T}_{*}(\vartheta, \gamma)-\mathscr{T}_{*}(u, \gamma) \geq \omega \Omega(\xi(\vartheta, u)), \\
& \mathscr{T}^{*}(\vartheta, \gamma)-\mathscr{T}^{*}(u, \gamma) \geq \omega \Omega(\xi(\vartheta, u)),
\end{aligned}
$$

that is

$$
\mathscr{T}(\vartheta) \sim \mathscr{T}(u) \geqslant \omega \Omega(\xi(\vartheta, u)) .
$$

This completes the proof.

Remark 4. If $\mathscr{T}$ is a G-differentiable strongly generalized preinvex fuzzy mapping modulus $\omega>0$, and

$$
\left\langle\mathscr{T}^{\prime}(u), \xi(\vartheta, u)\right\rangle \tilde{+} \omega \Omega(\xi(\vartheta, u)) \geqslant \widetilde{0},
$$

$\forall u, \vartheta \in K_{\xi}$, then $u \in K_{\xi}$ is the minimum of the mapping $\mathscr{T}$.

The inequality of the type in (120) is called strongly generalized fuzzy variational-like inequality. We would like to emphasize that the optimality conditions of the strongly generalized preinvex fuzzy mapping can be characterized by the following inequality:

$$
\left\langle\mathscr{T}^{\prime}(u), \xi(\vartheta, u)\right\rangle \geqslant \widetilde{0},
$$

and, $\forall u, \vartheta \in K_{\xi}$, this is called variational-like inequality.

We consider the functional $I(9)$, defined as

$$
I(\vartheta)=\mathscr{T}(\vartheta) \tilde{+} \mathscr{J}(\vartheta) \text {, }
$$

$\forall \vartheta \in \mathbb{R}$, where $\mathscr{T}$ is a G-differentiable preinvex fuzzy mapping and $\mathscr{F}$ is a non-G-differentiable strongly preinvex fuzzy mapping.

We show that the minimum of the functional $I(9)$ can be characterized by a class of variational-like inequalities.

Theorem 14. Let $\mathscr{T}: K_{\xi} \longrightarrow \mathbb{F}_{0}$ be a G-differentiable preinvex fuzzy mapping and let $\mathscr{f}: K_{\xi} \longrightarrow \mathbb{F}_{0}$ be a non-G-differentiable strongly generalized preinvex fuzzy mapping. Then the functional $I(\vartheta)$ has minimum $u \in K_{\xi}$ if and only if $u \in K_{\xi}$ satisfies

$$
\left\langle\mathscr{T}^{\prime}(u), \xi(\vartheta, u)\right\rangle \tilde{+} \mathscr{J}(\vartheta) \tilde{-} \mathscr{J}(u) \geqslant \omega \Omega(\xi(\vartheta, u)), \quad \forall \vartheta \in K_{\xi} .
$$

Proof. Let $u \in K_{\xi}$ be the minimum of $I$; then, by definition, for all $\vartheta \in K_{\xi}$, we have

$$
I(u) \preccurlyeq I(9),
$$

and since $K_{\xi}$ is an invex set, $\vartheta_{\tau}=u+\tau \xi(\vartheta, u)$, for all $u, \vartheta \in K_{\xi}$ and $\tau \in[0,1]$. Replacing $\vartheta$ by $\vartheta_{\tau}$ in (124), we get

$$
I(u) \preccurlyeq I(u+\tau \xi(\vartheta, u)),
$$

and, therefore, for every $\gamma \in[0,1]$, we have

$$
\begin{aligned}
& I_{*}(u, \gamma) \leq I_{*}(u+\tau \xi(\vartheta, u), \gamma), \\
& I^{*}(u, \gamma) \leq I^{*}(u+\tau \xi(\vartheta, u), \gamma) .
\end{aligned}
$$

which implies that, using (122),

$$
\begin{aligned}
& \mathscr{T}_{*}(u, \gamma)+\mathscr{J}_{*}(u, \gamma) \leq \mathscr{T}_{*}(u+\tau \xi(\vartheta, u), \gamma)+\mathscr{J}_{*}(u+\tau \xi(\vartheta, u), \gamma), \\
& \mathscr{T}^{*}(u, \gamma)+\mathscr{J}^{*}(u, \gamma) \leq \mathscr{T}^{*}(u+\tau \xi(\vartheta, u), \gamma)+\mathscr{J}^{*}(u+\tau \xi(\vartheta, u), \gamma) .
\end{aligned}
$$

Since $\mathscr{J}$ is a strongly generalized preinvex fuzzy mapping,

$$
\begin{aligned}
0 \leq & \mathscr{T}_{*}(u+\tau \xi(\vartheta, u), \gamma)-\mathscr{T}_{*}(u, \gamma) \\
& +\tau\left(\mathscr{J}_{*}(\vartheta, \gamma)-\mathscr{J}_{*}(u, \gamma)\right)-\omega \tau(1-\tau) \Omega(\xi(\vartheta, u)), \\
0 \leq & \mathscr{T}^{*}(u+\tau \xi(\vartheta, u), \gamma)-\mathscr{T}^{*}(u, \gamma) \\
& +\tau\left(\mathscr{J}^{*}(\vartheta, \gamma)-\mathscr{J}^{*}(u, \gamma)\right)-\omega \tau(1-\tau) \Omega(\xi(\vartheta, u)) .
\end{aligned}
$$

Now, dividing by " $\tau$ " and taking $\lim _{\tau \longrightarrow 0}$, we have 


$$
\begin{aligned}
& 0 \leq \lim _{\tau \longrightarrow 0}\left\{\frac{\mathscr{T}_{*}(u+\tau \xi(\vartheta, u), \gamma)-\mathscr{T}_{*}(u, \gamma)}{\tau}+\mathscr{J}_{*}(\vartheta, \gamma)-\mathscr{J}_{*}(u, \gamma)-\omega(1-\tau) \Omega(\xi(\vartheta, u))\right\}, \\
& 0 \leq \lim _{\tau \longrightarrow 0}\left\{\frac{\mathscr{T}^{*}(u+\tau \xi(\vartheta, u), \gamma)-\mathscr{T}^{*}(u, \gamma)}{\tau}+\mathscr{J}^{*}(\vartheta, \gamma)-\mathscr{J}^{*}(u, \gamma)-\omega(1-\tau) \Omega(\xi(\vartheta, u))\right\},
\end{aligned}
$$

Since $\lim _{\tau \longrightarrow 0}\left(\mathscr{T}_{*}(u+\tau \xi(\vartheta, u), \gamma)-\mathscr{T}_{*}(u, \gamma) / \tau\right)=\left\langle\mathscr{T}_{*}\right.$, $(u, \gamma), \xi(\vartheta, u)\rangle$ and $\lim _{\tau \longrightarrow 0}\left(\mathscr{T}^{*}(u+\tau \xi(\vartheta, u), \gamma)-\mathscr{T}^{*}(u\right.$, $\gamma) / \tau)=\left\langle\mathscr{T}^{*}(u, \gamma), \xi(\vartheta, u)\right\rangle$, from the above inequalities, we have

$$
\begin{aligned}
& 0 \leq\left\langle\mathscr{T}_{*}^{\prime}(u, \gamma), \xi(\vartheta, u)\right\rangle+\mathscr{F}_{*}(\vartheta, \gamma)-\mathscr{F}_{*}(u, \gamma)-\omega \Omega(\xi(\vartheta, u)), \\
& 0 \leq\left\langle\mathscr{T}^{*},(u, \gamma), \xi(\vartheta, u)\right\rangle+\mathscr{J}^{*}(\vartheta, \gamma)-\mathscr{J}^{*}(u, \gamma)-\omega \Omega(\xi(\vartheta, u)),
\end{aligned}
$$

Since $\mathscr{T}$ is a fuzzy mapping, from the above inequalities, we have

$$
\widetilde{0} \preccurlyeq\left\langle\mathscr{T}^{\prime}(u), \xi(\vartheta, u)\right\rangle \tilde{+} \mathscr{J}(\vartheta) \sim \mathcal{L}(u) \sim \omega \Omega(\xi(\vartheta, u)) .
$$

Conversely, let (123) be satisfied to prove that $u \in K_{\xi}$ is a minimum of $I$. Assume that, for all $\vartheta \in K_{\xi}$, we have

$$
I(u) \simeq I(\vartheta)=\mathscr{T}(u) \tilde{+} \mathscr{J}(u) \sim \mathscr{T}(\vartheta) \simeq \mathscr{J}(\vartheta) .
$$

Therefore, for every $\gamma \in[0,1]$, we have

$$
\begin{aligned}
& I_{*}(u, \gamma)-I_{*}(\vartheta, \gamma)=\mathscr{T}_{*}(u, \gamma)-\mathscr{T}_{*}(\vartheta, \gamma)+\mathscr{J}_{*}(u, \gamma)-\mathscr{J}_{*}(\vartheta, \gamma), \\
& I^{*}(u, \gamma)-I^{*}(\vartheta, \gamma)=\mathscr{T}^{*}(u, \gamma)-\mathscr{T}^{*}(\vartheta, \gamma)+\mathscr{J}^{*}(u, \gamma)-\mathscr{J}^{*}(\vartheta, \gamma) .
\end{aligned}
$$

By Theorem 7, we have

$$
\begin{aligned}
& I_{*}(u, \gamma)-I_{*}(\vartheta, \gamma) \leq-\left[\left\langle\mathscr{T}_{*}^{\prime}(u, \gamma), \xi(\vartheta, u)\right\rangle+\mathscr{J}_{*}(\vartheta, \gamma)-\mathscr{J}_{*}(u, \gamma)\right], \\
& I^{*}(u, \gamma)-I^{*}(\vartheta, \gamma) \leq-\left[\left\langle\mathscr{T}^{*}(u, \gamma), \xi(\vartheta, u)\right\rangle+\mathscr{J}^{*}(\vartheta, \gamma)-\mathscr{J}^{*}(u, \gamma)\right] .
\end{aligned}
$$

By using (123), we have

$$
\begin{aligned}
& I_{*}(u, \gamma)-I_{*}(\vartheta, \gamma) \leq-\omega \Omega(\xi(\vartheta, u)) \leq 0, \\
& I^{*}(u, \gamma)-I^{*}(\vartheta, \gamma) \leq-\omega \Omega(\xi(\vartheta, u)) \leq 0,
\end{aligned}
$$

and, hence, $I(u) \preccurlyeq I(\vartheta)$.

Note that (123) is called a strongly generalized fuzzy mixed variational-like inequality. This result shows the characterization of the minimum of strongly generalized preinvex fuzzy mapping with the help of strongly generalized fuzzy mixed variational-like inequalities.

Now, we discuss some special cases of strongly generalized fuzzy mixed variational-like inequalities:

(i) If $\Omega \xi((\vartheta, u))=\|\xi(\vartheta, u)\|^{2}$, then (123) is called a strongly fuzzy mixed variational-like inequality such as

$$
\left\langle\mathscr{T}^{\prime}(u), \xi(\vartheta, u)\right\rangle \tilde{+} \mathscr{J}(\vartheta) \sim \mathcal{F}(u) \tilde{\sim} \omega\|(\vartheta, u)\|^{2} \geqslant \widetilde{0}, \quad \forall \vartheta \in K_{\xi} .
$$

(ii) If $\xi(\vartheta, u)=\vartheta-u$, then (136) is called a strongly fuzzy mixed variational inequality such as

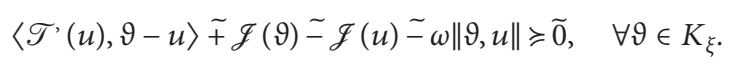

(iii) If $\omega=0$, then (136) is called a fuzzy mixed variational-like inequality such as

$$
\left\langle\mathscr{T}^{\prime}(u), \xi(\vartheta, u)\right\rangle \tilde{+} \mathscr{J}(\vartheta) \sim \mathcal{J}(u) \geqslant \widetilde{0}, \quad \forall \vartheta \in K_{\xi} .
$$

(iv) If $\omega=0$, then (137) is called a fuzzy mixed variational inequality such as

$$
\left\langle\mathscr{T}^{\prime}(u), \vartheta-u\right\rangle \tilde{+} \mathscr{J}(\vartheta) \sim \mathscr{J}(u) \geqslant \widetilde{0}, \quad \forall \vartheta \in K_{\xi} .
$$

(v) If $\mathscr{J}=0$, then (138) is known as a fuzzy variationallike inequality.

$$
\left\langle\mathscr{T}^{\prime}(u), \xi(\vartheta, u)\right\rangle \geqslant \widetilde{0}, \quad \forall \vartheta \in K_{\xi} .
$$

(vi) If $\mathscr{J}=0$, then (139) is known as a fuzzy variational inequality.

$$
\left\langle\mathscr{T}^{\prime}(u), \vartheta-u\right\rangle \geqslant \widetilde{0}, \quad \forall \vartheta \in K_{\xi}
$$

Theorem 15. Let $\mathscr{T}: K_{\xi} \longrightarrow \mathbb{F}_{0}$ be a G-differentiable invex fuzzy mapping and let $\mathscr{J}: K_{\xi} \longrightarrow \mathbb{F}_{0}$ be a non-G-differentiable strongly preinvex fuzzy mapping. Then functional $I(\vartheta)$ has minimum $u \in K_{\xi}$ if and only if $u \in K_{\xi}$ satisfies

$$
\left\langle\mathscr{T}^{\prime}(u), \xi(\vartheta, u)\right\rangle \tilde{+} \mathscr{J}(\vartheta) \sim \mathscr{L}(u) \sim \omega\left(\Omega(\xi(\vartheta, u)) \geqslant \widetilde{0}, \quad \forall \vartheta \in K_{\xi} .\right.
$$

Proof. The proof is similar to that of Theorem 14; it is omitted here.

Remark 5. Inequality (123) shows that the variational-like inequalities arise naturally in connection with the minimization of the G-differentiable preinvex fuzzy mappings subject to certain constraints.

\section{Conclusion}

In this paper, we introduced and studied a new class of fuzzy nonconvex mappings with respect to nonnegative function and bifunction known as strongly generalized preinvex fuzzy mappings. We proved that convex and preinvex fuzzy mappings are special cases of strongly generalized preinvex fuzzy mappings. In case of G-differentiable preinvex fuzzy mappings and non-G-differential strongly generalized preinvex fuzzy mappings, we introduced the new class of fuzzy variational-like inequalities is known as strongly generalized 
fuzzy mixed variational-like inequalities characterizing the optimality conditions for the sum of G-differentiable preinvex fuzzy mappings and non-G-differentiable strongly generalized preinvex fuzzy mappings. We hope that this link between these fields may lead to significant, new, and innovative result.

\section{Data Availability}

No data were used to support this study.

\section{Conflicts of Interest}

The authors declare that they have no conflicts of interest.

\section{Authors' Contributions}

All authors contributed equally to the writing of this paper and all of them read and approved the final manuscript.

\section{Acknowledgments}

The authors acknowledge the Rector, COMSATS University Islamabad, Islamabad, Pakistan, for providing excellent research and academic environments. This study was funded by the National Natural Science Foundation of China (no. 71771140).

\section{References}

[1] M. A. Hanson, "On sufficiency of the Kuhn-Tucker conditions," Journal of Mathematical Analysis and Applications, vol. 80 , pp. 545-550, 1980.

[2] A. Ben-Isreal and B. Mond, "What is invexity," The Anziam Journal, vol. 28, pp. 1-9, 1986.

[3] S. R. Mohan and S. K. Neogy, "On invex sets and preinvex functions," Journal of Mathematical Analysis and Applications, vol. 189, no. 3, pp. 901-908, 1995.

[4] M. A. Noor, "Generalized convex functions," Panamerican Mathematical Journal, vol. 4, pp. 73-89, 1994.

[5] T. Weir and B. Mond, "Preinvex functions in multiobjective optimization," Journal of Mathematical Analysis and Applications, vol. 136, pp. 29-38, 1986.

[6] M. A. Noor, "Variational-like inequalities," Optimization, vol. 30, pp. 323-330, 1994.

[7] M. A. Noor and K. I. Noor, "On strongly generalized preinvex functions," Journal of Inequalities in Pure and Applied Mathematics, vol. 6, p. 102, 2005.

[8] M. A. Noor and K. I. Noor, "Some characterizations of strongly preinvex functions," Journal of Mathematical Analysis and Applications, vol. 316, no. 2, pp. 697-706, 2006.

[9] B. T. Polyak, "Existence theorems and convergence of minimizing sequences in extremum problems with restrictions," Soviet Mathematics Doklady, vol. 7, pp. 2-75, 1966.

[10] S. Karamardian, "The nonlinear complementarity problem with applications, Part 2," Journal of Optimization Theory and Applications, vol. 4, no. 3, pp. 167-181, 1969.

[11] G. Qu and N. Li, "On the exponential stability of primal-dual gradient dynamics," IEEE Control Systems Letters, vol. 3, no. 1, pp. 43-48, 2019.

[12] K. Nikodem and Z. Pales, "Characterizations of inner product spaces by strongly convex functions," Banach Journal of Mathematical Analysis, vol. 5, no. 1, pp. 83-87, 2011.
[13] M. Adamek, "On a problem connected with strongly convex functions," Mathematical Inequalities \& Applications, vol. 19, no. 4, pp. 1287-1293, 2016.

[14] N. I. Akhiezer, The Classical Moment Problem and Some Related Questions in Analysis, Oliver and Boyd, Edinburgh, Scotland, 1965.

[15] M. U. Awan, M. A. Noor, V. N. Mishra, and K. I. Noor, "Some characterizations of general preinvex functions," International Journal of Analysis and Applications, vol. 15, pp. 46-56, 2017.

[16] M. V. Jovanovic, "A note on strongly convex and strongly quasiconvex functions," Mathematics Notes, vol. 60, pp. 584-585, 1966.

[17] Z.-Y. Peng, X.-J. Long, and Z. Lin, "Some new properties of strongly convex fuzzy sets," Advances in Intelligent and Soft Computing, vol. 2, pp. 687-693, 2009.

[18] A. Iqbal and I. Ahmad, "Strong geodesic convex functions of order m," Numerical Functional Analysis and Optimization, vol. 40, no. 15, pp. 1840-1846, 2019.

[19] M. A. Khan, I. Ahmad, and A. Aljohani, "Criterion for generalized weakly fuzzy invex monotonocities," Advances in Fuzzy Systems, vol. 2018, Article ID 3749650, 2018.

[20] Z. Y. Peng, "Semistrict, G-preinvexity and its application," Journal of Inequalities and Applications, vol. 198, no. 1, pp. 1-11, 2012.

[21] Z. Y. Peng and S. S. Chang, "Some properties of semi-Gpreinvex functions," Taiwanese Journal of Mathematics, vol. 17, no. 3, pp. 873-884, 2013.

[22] Z.-Y. Peng, K.-K. Li, and J.-T. Zhou, "Semi-prequasi-invex type multiobjective optimization and generalized fractional programming problems," Journal of Nonlinear Sciences and Applications, vol. 9, no. 12, pp. 6142-6152, 2016.

[23] M. B. Khan, M. A. Noor, K. I. Noor, A. T. Ab Ghani, and L. Abdullah, "Extended perturbed mixed variational-like inequalities for fuzzy mappings," Journal of Mathematics, vol. 2021, Article ID 6652930, 2021.

[24] L. A. Zadeh, "Fuzzy sets," Information and Control, vol. 8, no. 3, pp. 338-353, 1965.

[25] Y.-M. Liu, "Some properties of convex fuzzy sets," Journal of Mathematical Analysis and Applications, vol. 111, no. 1, pp. 119-129, 1985.

[26] R. Lowen, "Convex fuzzy sets," Fuzzy Sets and Systems, vol. 3, no. 3, pp. 291-310, 1980.

[27] E. Ammar and J. Metz, "On fuzzy convexity and parametric fuzzy optimization,” Fuzzy Sets and Systems, vol. 49, no. 2, pp. 135-141, 1992.

[28] A. K. Katsaras and D. B. Liu, "Fuzzy vector spaces and fuzzy topological vector spaces," Journal of Mathematical Analysis and Applications, vol. 58, no. 1, pp. 135-146, 1977.

[29] M. A. Noor and K. I. Noor, "Generalized preinvex functions and their properties," International Journal of Stochastic Analysis, vol. 2006, p. 12736, 2006.

[30] D. Dubois and H. Prade, "Operations on fuzzy numbers," International Journal of Systems Science, vol. 9, no. 6, pp. 613-626, 1978.

[31] R. Goetschel and W. Voxman, "Topological properties of fuzzy numbers," Fuzzy Sets and Systems, vol. 10, no. 1-3, pp. 87-99, 1983.

[32] N. Furukawa, "Convexity and local Lipschitz continuity of fuzzy-valued mappings," Fuzzy Sets and Systems, vol. 93, no. 1, pp. 113-119, 1998.

[33] S. Nanda and K. Kar, "Convex fuzzy mappings," Fuzzy Sets and Systems, vol. 48, no. 1, pp. 129-132, 1992. 
[34] Y.-R. Syau, "On convex and concave fuzzy mappings," Fuzzy Sets and Systems, vol. 103, no. 1, pp. 163-168, 1999.

[35] R. Goetschel and W. Voxman, "Elementary fuzzy calculus," Fuzzy Sets and Systems, vol. 18, no. 1, pp. 31-43, 1986.

[36] H. Yan and J. Xu, "A class of convex fuzzy mappings," Fuzzy Sets and Systems, vol. 129, no. 1, pp. 47-56, 2002.

[37] M. A. Noor, "Fuzzy preinvex functions," Fuzzy Sets and Systems, vol. 64, no. 1, pp. 95-104, 1994.

[38] Y.-R. Syau, "Preinvex fuzzy mappings," Computers \& Mathematics with Applications, vol. 37, no. 3, pp. 31-39, 1999.

[39] Y.-R. Syau and E. S. Lee, "Fuzzy Weirstrass theorem and convex fuzzy mappings," Computers \& Mathematics with Applications, vol. 51, no. 12, pp. 1741-1750, 2006.

[40] B. Bede and S. G. Gal, "Generalizations of the differentiability of fuzzy-number-valued functions with applications to fuzzy differential equations," Fuzzy Sets and Systems, vol. 151, no. 3, pp. 581-599, 2005.

[41] X. P. Ding and J. Y. Park, "A new class of generalized nonlinear implicit quasivariational inclusions with fuzzy mappings," Journal of Computational and Applied Mathematics, vol. 138, no. 2, pp. 243-257, 2002.

[42] J. Li and M. A. Noor, "On characterizations of preinvex fuzzy mappings," Computers \& Mathematics with Applications, vol. 59, no. 2, pp. 933-940, 2010.

[43] L. Stefanini and B. Bede, "Generalized Hukuhara differentiability of interval-valued functions and interval differential equations," Nonlinear Analysis: Theory, Methods \& Applications, vol. 71, no. 3-4, pp. 1311-1328, 2009. 